\title{
Activités biologiques de Tetraclinis articulata : revue de synthèse
}

\author{
Ilham ZAHIR $^{1 *}$, Asma ER-RAHMANY ${ }^{1}$, Raja Es-SADOUNY ${ }^{1}$, Issam El HADRI ${ }^{1}$ \\ Manuscrit reçu le 7 juin 2020 et accepté le 3 août 2020
}

1 Laboratoire Polyvalent en Recherche et Développement, Département de Biologie. Université Sultan Moulay Slimane. Faculté Polydisciplinaire Béni Mellal. Mghila, Béni Mellal, 23030, Maroc

\section{Résumé}

Dans le cadre de la mise en valeur des vertus de la plante Tetraclinis articulata, une espèce considérée comme très importante en Afrique du Nord et connue sous le nom de thuya de barbarie, une revue de synthèse a été menée à partir de l'analyse d'un ensemble de recherches scientifiques réalisées principalement au Maghreb et en Égypte et abordant les principales activités biologiques de cette essence forestière.

En fait, il s'agit d'une plante très employée dans la médecine populaire. Incontestablement, elle est préconisée aussi bien pour le traitement des infections intestinales et respiratoires que pour le diabète, l'hypertension et la fièvre. Par ailleurs, la plupart des études phytochimiques des huiles essentielles et/ou des extraits des différentes parties du thuya montrent que cette plante est riche en composants majeurs tels que les flavonoïdes, les polyphénols et les terpènes. Une ou plusieurs de ces molécules pourraient être la raison pour laquelle l'espèce $T$. articulata est dotée de maints pouvoirs biologiques, à savoir les activités antimicrobiennes (antibactériennes et antifongiques), anti-inflammatoires et antioxydantes, ce qui fait de la plante en question un immense réservoir à exploiter dans les domaines médical, alimentaire, cosmétique et agricole. Bien que l'espèce $T$. articulata soit caractérisée par une faible toxicité aigüe, des études toxicologiques pertinentes des extraits et/ou des huiles essentielles du thuya doivent être envisagées afin de juger de l'innocuité de cet arbre aussi bien pour l'environnement que pour l'organisme humain.

Mots clés : Tetraclinis articulata, huiles essentielles, extraits, activité antioxydante, activité anti-inflammatoire, activité antimicrobienne, principes actifs.

\begin{abstract}
To highlight the virtues of Tetraclinis articulata, a very important species in North Africa and known under the name of barbary thuja, a review was undertaken from the analysis of scientific investigations mainly carried out in the Maghreb and Egypt and which focused on the main biological activities of this forest species.

In fact, it is a plant widely used in folk medicine. Undoubtedly, it is recommended for the treatment of intestinal and respiratory infections as well as for diabetes, hypertension and fever. Furthermore, most phytochemical studies of essential oils and / or extracts of different parts of thuja show that this plant is rich in major components such as flavonoids, polyphenols and terpenes. One or more of these molecules could be the reason why $T$. articulata is endowed with many biological effects, namely antimicrobial (antibacterial and antifungal), anti-inflammatory and antioxidant activities. That makes of the plant in question an immense reservoir to explore in the medical, food, cosmetic and agricultural fields.
\end{abstract}

\footnotetext{
* Adresse pour le courrier électronique : ilham.zahir@usms.ma
} 
Although T. articulata is characterized by a low acute toxicity, relevant toxicological studies of thuja extracts and / or essential oils have to be performed to judge the safety of this tree as well for the environment and for the human organism.

Keywords : Tetraclinis articulata, essential oils, extracts, antioxidant activity, antiinflammatory activity, antimicrobial activity, active components.

\section{Introduction}

L'espèce Tetraclinis articulata, connue sous le nom de thuya de barbarie, est un arbre monoïque qui appartient à l'embranchement des Gymnospermes et à la famille des Cupressacées (Hadjadj et Letreuch Belarouci, 2017 ; Zahir et Rahmani, 2020) et qui, essentiellement limité à la région méditerranéenne méridionale occidentale (Hadjadj et Letreuch Belarouci, 2017), constitue un élément important dans la végétation forestière nordafricaine.

Cet arbre joue un rôle socio-économique important dans le domaine de l'artisanat, et ce, grâce à son bois de grande valeur employé en ébénisterie et à son écorce riche en résine dont les extraits sont utilisés dans la fabrication de certains vernis (Montassir et al., 2017 ; Mhirit et Benchekroun, 2006).

Par ailleurs, cette essence forestière est très utilisée en médecine traditionnelle en raison de ses multiples effets thérapeutiques. En effet, différentes parties du thuya sont préconisées dans le traitement des infections intestinales, des douleurs gastriques, des maladies respiratoires, du diabète, de l'hypertension et de la fièvre (Zahir et Rahmani, 2020). Ces utilisations révèlent que les extraits et/ou les huiles essentielles (HE) de T. articulata possèdent des composés actifs qui exercent de nombreuses activités biologiques (Dane et al., 2015 ; El Jemli et al., 2016 a ; Montassir et al., 2017 ; Rached et al., 2018 ; Sadiki et al., 2018).

C'est dans ce sens que cette revue de synthèse revêt un intérêt capital. Elle consiste à mettre en valeur les effets biologiques du thuya, et parmi celles-ci, les activités antimicrobiennes, antioxydantes, anti-inflammatoires et antitumorales, ainsi que l'effet larvicide. Ce travail aborde également les résultats de recherches traitant de la toxicité de cette plante, et ce, afin d'aviser le public de ses limites d'utilisation dans la médecine traditionnelle et moderne et de souligner le danger éventuel de son emploi et de ses retombées sur les espèces-clés des écosystèmes aquatiques et terrestres. 


\section{Méthodologie}

Afin de relever les principales activités de T. articulata, des articles scientifiques ont été analysés après avoir été recensés à partir de diverses bases de données, notamment Science Direct, Pubmed, Scopus, Google Scholars et Google.

Les publications retenues ont été filtrées en fonction du titre et du résumé ou des deux en même temps. Il est à noter ici que les articles ainsi sélectionnés répondent à la thématique abordée et sont tous rédigés en français ou en anglais. Pour chacune des recherches sélectionnées, les informations suivantes ont été identifiées et rassemblées :

* La région de collecte de la plante objet de la recherche ;

* Les parties utilisées pour l'obtention des extraits et/ou des HE du végétal ;

* Les valeurs des diamètres d'inhibition des extraits et/ou des HE vis-à-vis des bactéries, ainsi que celles des concentrations minimales inhibitrices (CMI) et bactéricides (CMB), avec enregistrement de la méthodologie adoptée ;

* Le pourcentage d'inhibition de la croissance mycélienne par extraits et/ou HE de thuya de barbarie, ainsi que leurs CMI et leurs concentrations minimales fongicides (CMF) ;

* Les modèles expérimentaux utilisés pour mettre en évidence les activités antioxydantes et anti-inflammatoires de la plante en question, ainsi que les résultats obtenus ;

* Les composés majeurs ou actifs des parties utilisées de T. articulata, avec détermination des techniques exécutées ;

* La toxicité aigüe éventuelle des extraits et/ou des HE de la plante.

\section{Résultats et discussion}

\subsection{Propriétés pharmacologiques}

\subsubsection{Activité antimicrobienne}

\subsubsection{Activité antibactérienne}

La résistance bactérienne aux antibiotiques représente un véritable problème de santé publique au monde. En effet, ces dernières décennies, le risque de contracter des maladies infectieuses s'est sensiblement accru en raison de l'émergence et de la propagation de bactéries multi-résistantes (Zahir et al., 2018 a). Devant cette situation, plusieurs investigations ont été menées pour la découverte de principes actifs à effet antibactérien à 
partir des plantes. C'est dans ce sens que l'activité antibactérienne de l'HE extraite par hydrodistillation à partir du bois de la loupe de T. articulata de la région d'Oulmès (Maroc) a été évaluée contre quatre bactéries, à savoir : Enterococcus faecalis, Escherichia coli, Klebsiella pneumoniae et Streptococcus D. Ainsi, des effets inhibiteurs considérables contre toutes les bactéries soumises au test ont été observés avec des CMI et des CMB proches de 1,25 $\mu \mathrm{L} / \mathrm{mL}$. Cette activité pourrait être due à un ou à plusieurs des quinze composés détectés par l'analyse de l'HE. Il est à signaler que cette analyse, réalisée grâce à la chromatographie en phase gazeuse couplée à la spectrométrie de masse (CPG-SM), révèle des composés majoritaires comme le cédrène (23\%), le thymol (22\%), le 3-tert-butyl-4-méthoxyphénol (19\%), le cédrénol $(9,6 \%)$, le muurolène $(5,4 \%)$ et le sclarène $(3 \%)$. Les résultats de cette étude suggèrent que l'HE du bois de la loupe de T. articulata a le potentiel d'être utilisée comme un agent bactéricide (El Moussaouiti et al., 2010).

Une autre étude a été effectuée sur des feuilles de $T$. articulata collectées dans la forêt de Ghazaouet dans la région de Tlemcen (Algérie). L'activité antibactérienne in vitro de l'HE extraite par hydro-distillation des feuilles séchées a été évaluée et elle a révélé un effet notable contre des bactéries du type Staphylococcus aureus ATCC 25923, Bacillus cereus ATCC11778, Pseudomonas aeruginosa ATCC27853, Escherichia coli ATCC 25922 et E. coli ATCC25921 (ABI-Ayad et al., 2011).

Des investigations supplémentaires ont démontré également l'effet antibactérien d'extraits et/ou d'HE obtenus à partir d'autres parties du thuya de barbarie comme le montre le tableau 1.

Tableau 1 : Activité antibactérienne de Tetraclinis articulata

\begin{tabular}{|c|c|c|c|c|c|c|c|}
\hline Région & $\begin{array}{l}\text { Partie } \\
\text { utilisée }\end{array}$ & Extrait/ HE * & $\begin{array}{c}\text { Activité contre les } \\
\text { bactérie (s) sensible (s) }\end{array}$ & CMI & СМВ & $\begin{array}{l}\text { Méthode (s) } \\
\text { utilisée (s) }\end{array}$ & $\begin{array}{l}\text { Composé (s) } \\
\text { majeur (s) ou } \\
\text { actif (s) }\end{array}$ \\
\hline $\begin{array}{l}\text { Khémisset } \\
\text { (Maroc) } \\
\text { (Boukhriss } \\
\text { et al., 2007) }\end{array}$ & Feuilles & $\begin{array}{l}\text { L'HE a été } \\
\text { extraite par } \\
\text { hydro- } \\
\text { distillation. }\end{array}$ & $\begin{array}{l}\text { Micrococcus luteus } \\
\text { Bacillus subtilis } \\
\text { Staphylococcus aureus } \\
\text { Escherichia coli }\end{array}$ & $\begin{array}{l}1 / 1000 \mathrm{~V} / \mathrm{V} \\
1 / 500 \\
\mathrm{~V} / \mathrm{V} \quad 1 / 5000 \\
\mathrm{~V} / \mathrm{V} 1 / 500 \\
\mathrm{~V} / \mathrm{V}\end{array}$ & ND & $\begin{array}{l}\text { Différentes } \\
\text { concentrations } \\
\text { d'HE ont été } \\
\text { incorporées } \\
\text { dans le milieu } \\
\text { nutritif solide. }\end{array}$ & $\begin{array}{l}\text { Les principaux } \\
\text { composés de l'HE } \\
\text { ont été identifiés } \\
\text { par CPG et } \\
\text { CPG/SM : } \\
\text { l'acétate de } \\
\text { bornyle }(30,74 \%) \text {, } \\
\text { l' } \alpha \text {-pinène } \\
(23,54 \%), \text { le } \\
\text { camphre }(17,27 \%) \\
\text { et le limonène } \\
(23,31 \%) .\end{array}$ \\
\hline
\end{tabular}


Bulletin de la Société Royale des Sciences de Liège, Vol. 89, articles, 2020, p. 91 - 114

\begin{tabular}{|c|c|c|c|c|c|c|c|}
\hline $\begin{array}{l}\text { Hammam } \\
\text { Melouane } \\
(\mathrm{H}) \text { et } \\
\text { Tipaza (T) } \\
\text { (Algérie) } \\
\text { (40 km au } \\
\text { sud et } \\
70 \text { km à } \\
\text { l'ouest) } \\
\text { (Chikhoune } \\
\text { et al., 2013) }\end{array}$ & $\begin{array}{l}\text { Feuilles et } \\
\text { cônes }\end{array}$ & $\begin{array}{l}\text { L'HE a été } \\
\text { extraite par } \\
\text { hydro- } \\
\text { distillation. }\end{array}$ & $\begin{array}{l}\text { Escherichia coli: } \\
\text { * Les DAI de l'HEF des } \\
\text { régions H et T sont de } \\
\text { l'ordre de: } 7 \pm 0,1 \mathrm{~mm} ; \\
10 \pm 0,1 \\
\text { respectivement. } \\
\text { * Les DAI de l'HEC } \\
\text { des régions H et T } \\
\text { sont de l'ordre de : } 10 \pm \\
0,2 \mathrm{~mm} ; 9 \pm 0,1 \mathrm{~mm} \text {, } \\
\text { respectivement. } \\
\text { Pseudomonas } \\
\text { aeruginosa : } \\
\text { * Les DAI de l'HEF des } \\
\text { régions H et T sont de } \\
\text { l'ordre de : 7,5 } \pm 0,2 \\
\text { mm ; } 11 \pm 0,2 \mathrm{~mm}, \\
\text { respectivement. } \\
\text { * Les DAI de l'HEC } \\
\text { des régions H et T } \\
\text { sont de l'ordre de : } 12 \pm \\
0,2 \text { mm ; } 9,5 \pm 0,1 \\
\text { respectivement } \\
\text { Staphylococcus aureus } \\
* \text { Les DAI de l'HEF des } \\
\text { régions H et T sont de } \\
\text { l'ordre de : } 22 \pm 0,9 \\
\text { mm; } 18 \pm 0,4 \text { mm, } \\
\text { respectivement } \\
\text { * Les DAI de l'HEC } \\
\text { des régions H et T } \\
\text { sont de l'ordre de }: 17 \pm \\
0,6 \text { mm ; } 15 \pm 0,2 \mathrm{~mm}, \\
\text { respectivement. }\end{array}$ & $\begin{array}{l}\text { } \\
0,4 \mu \mathrm{g} / \mathrm{mL}(\mathrm{H}) \\
\mathrm{ND}(\mathrm{T}) \\
0,2 \mu \mathrm{g} / \mathrm{mL} \mathrm{H}) \\
0,2 \mu \mathrm{g} / \mathrm{mL}(\mathrm{T})\end{array}$ & ND & $\begin{array}{l}\text { Méthode de } \\
\text { diffusion sur } \\
\text { disques (AAB) } \\
\text { Technique de } \\
\text { dilution en } \\
\text { milieu solide } \\
\text { (CMI) }\end{array}$ & $\begin{array}{l}\text { Les composants } \\
\text { principaux des HE } \\
\text { ont été extrait par } \\
\text { CPG et CPG / } \\
\text { SM : l' } \alpha \text {-pinène } \\
(19,8-24,9 \%) \text { et } \\
\text { l'acétate de } \\
\text { bornyle }(40,2- \\
59,2 \%) \text { pour les } \\
\text { feuilles. } \\
\text { L' } \alpha \text {-pinène } \\
(57,5-75 \%), \text { le } \\
\text { limonène }(10,6- \\
20,9 \%) \text { et le } \beta- \\
\text { myrcène }(3,6- \\
10,6 \%) \text { pour les } \\
\text { cônes. }\end{array}$ \\
\hline $\begin{array}{l}\text { Essaouira } \\
\text { (Maroc) } \\
\text { (Akbli et } \\
\text { al., 2016) }\end{array}$ & $\begin{array}{l}\text { Sciure du } \\
\text { bois de la } \\
\text { loupe }\end{array}$ & $\begin{array}{l}\text { L'HE a été } \\
\text { extraite par } \\
\text { hydro- } \\
\text { distillation. }\end{array}$ & $\begin{array}{l}\text { Escherichia coli } \\
\text { ATCC est extrêmement } \\
\text { sensible à } 5 \mu \mathrm{L} \text { de l'HE } \\
(\mathrm{DAI}=22,33 \pm 3,05 \\
\mathrm{mm}) \\
\text { E. coli clinique est } \\
\text { sensible à } 5 \mu \mathrm{L} \text { de l'HE } \\
\text { (DAI }=12 \pm 1 \mathrm{~mm}) \\
\text { Staphylococcus aureus } \\
\text { ATCC et } S \text {. aureus } \\
\text { clinique sont } \\
\text { extrêmement sensibles à } \\
5 \mu \mathrm{L} \text { de l'HE (DAI sont } \\
\text { de l'ordre de } 21 \pm 2,64 \\
\text { mm et } 20 \pm 1 \mathrm{~mm} \\
\text { respectivement) }\end{array}$ & ND & ND & $\begin{array}{l}\text { Méthode de } \\
\text { diffusion sur } \\
\text { disque à l'aide } \\
\text { d'un } \\
\text { aromatogramme } \\
\text { (AAB) }\end{array}$ & ND \\
\hline $\begin{array}{l}\text { Ain Defla } \\
\text { (Algérie) } \\
\text { (200 km à } \\
\text { l'ouest) } \\
\text { (Djouahri et } \\
\text { al., 2016) }\end{array}$ & Feuilles & $\begin{array}{l}\text { L'HE a été } \\
\text { extraite par } \\
\text { hydro- } \\
\text { distillation. }\end{array}$ & $\begin{array}{l}\text { Salmonella enterica } \\
\mathrm{E} 32(\mathrm{DAI}=17,5 \pm 0,5 \\
\mathrm{mm}) \\
\text { Klebsiella pneumoniae } \\
\text { CIP8291 }(\mathrm{DAI}=11,5 \pm \\
0,3 \mathrm{~mm}) \\
\text { Listeria monocytogenes } \\
\text { CIP82110 (DAI }=17,5 \\
\pm 0,5 \mathrm{~mm}) \\
\text { Staphylococcus aureus } \\
\text { CIP7625 (DAI }=25,3 \pm \\
1,7 \mathrm{~mm}) \\
\text { Pseudomonas } \\
\text { aeruginosa } \text { CIPA22 } \\
(\mathrm{DAI}=15,5 \pm 0,5 \mathrm{~mm}) \\
\text { E. coli } \mathrm{ATTC} 10536 \\
(\mathrm{DAI}=15,5 \pm 0,7 \mathrm{~mm})\end{array}$ & $\begin{array}{l}1 \mu \mathrm{g} / \mathrm{mL} \\
50 \mu \mathrm{g} / \mathrm{mL} \\
1 \mu \mathrm{g} / \mathrm{mL} \\
0,25 \mu \mathrm{g} / \mathrm{mL} \\
30 \mu \mathrm{g} / \mathrm{mL} \\
30 \mu \mathrm{g} / \mathrm{mL}\end{array}$ & ND & $\begin{array}{l}\text { Méthode de } \\
\text { diffusion sur } \\
\text { disques (AAB) } \\
\text { Technique de } \\
\text { dilution en } \\
\text { milieu solide } \\
\text { (CMI) }\end{array}$ & $\begin{array}{l}\text { Les composants } \\
\text { majeurs analysés } \\
\text { par CPG et CPG- } \\
\text { SM sont : } \\
\text { l' } \alpha \text {-pinène } \\
(29,0 \%), \text { l'acétate } \\
\text { de bornyle } \\
(25,5 \%), \text { le } \\
\text { camphre }(16,6 \%) \\
\text { et le limonène } \\
(6,7 \%) \\
\text { représentant } \\
96,6 \% \text { du total de } \\
\text { l'HE. }\end{array}$ \\
\hline
\end{tabular}


Bulletin de la Société Royale des Sciences de Liège, Vol. 89, articles, 2020, p. 91 - 114

\begin{tabular}{|c|c|c|c|c|c|}
\hline $\begin{array}{l}\text { Mornag et } \\
\text { Gouvernorat } \\
\text { de Ben } \\
\text { Arous } \\
\text { (Nord-Est } \\
\text { de la } \\
\text { Tunisie) } \\
\text { (Jlizi et } \text { al., } \\
\text { 2018) }\end{array}$ & Résine & ND & $\begin{array}{l}\text { Enterococcus faecalis } \\
(14,5 \pm 1 \mathrm{~mm}) \text {, } \\
\text { Staphylococcus aureus } \\
(15,8 \pm 1 \mathrm{~mm}) \\
\text { Pseudomonas } \\
\text { aeruginosa } \\
(8,4 \pm 1 \mathrm{~mm}) \\
\text { Escherichia coli } \\
(10,5 \pm 1 \mathrm{~mm})\end{array}$ & ND & ND \\
\hline
\end{tabular}

Méthode de
diffusion sur
gélose en
utilisant le
milieu Mueller
Hinton (MHA)

\begin{tabular}{lll}
\hline Khemisset & Sciure du & L'HE a été \\
(Maroc) & bois de & extraite par \\
(Talbaoui et & loupe & $\begin{array}{l}\text { hydro- } \\
\text { distillation. }\end{array}$ \\
al., 2016 ) & &
\end{tabular}

$\begin{array}{lcl}\begin{array}{l}\text { Enterococcus faecalis } \\ \text { (DAI }=19 \mathrm{~mm})\end{array} & 5 \mu \mathrm{L} / \mathrm{mL} & 5 \\ \text { Escherichia coli } & 10 \mu \mathrm{L} / \mathrm{mL} & \mu \mathrm{L} / \mathrm{mL} \\ \text { (DAI }=10 \mathrm{~mm}) & & \mu \mathrm{L} / \mathrm{mlL} \\ \text { Klebsiella pneumoniae } & 10 \mu \mathrm{L} / \mathrm{mL} & 10 \\ \text { (DAI }=17 \mathrm{~mm} \text { ) } & & \mu \mathrm{L} / \mathrm{mL} \\ \text { Streptococcus } \mathrm{D} & 10 \mu \mathrm{L} / \mathrm{mL} & 10 \\ \text { (DAI }=13 \mathrm{~mm}) & & \mu \mathrm{L} / \mathrm{mL}\end{array}$

$\begin{array}{llll} & & \\ & & \\ & & \\ & & \\ & & \\ \text { Oran } & \text { Feuilles } & \text { Une } & \text { Enterococcus faecalis } \\ \text { l'Algérie) } & \text { extraction par } & \text { Listeria monocytogenes } \\ \text { al., 2018) } & \text { partage } & \text { SASM } \\ \text { liquide-liquide } & \text { SARM } \\ & \text { a été effectuée } & \text { Eschericia coli } \\ \text { pour obtenir } & \text { E. coli } \text { BLSE } \\ \text { les fractions } & \text { Klebsiella pneumoniae } \\ & \text { organiques de } & \text { K. pneumoniae BLSE } \\ \text { l'extrait } & \text { Morganella morganii } \\ \text { aqueux en } & \text { Pseudomonasaeruginos } \\ & \text { utilisant de } & \text { a } \\ \text { l'acétate } & \\ \text { d'éthyle et du } & \\ \text { butanol, } & \\ \text { successiveme } & \\ \text { nt. } & \\ & & \end{array}$
$5 \mathrm{mg} / \mathrm{mL}$
$5 \mathrm{mg} / \mathrm{mL}$
$0,625 \mathrm{mg} / \mathrm{mL}$
$0,625 \mathrm{mg} / \mathrm{mL}$
$5 \mathrm{mg} / \mathrm{mL}$
$5 \mathrm{mg} / \mathrm{mL}$
$5 \mathrm{mg} / \mathrm{mL}$
$5 \mathrm{mg} / \mathrm{mL}$
$10 \mathrm{mg} / \mathrm{mL}$
$10 \mathrm{mg} / \mathrm{mL}$
Uniquement
les résultats de
la fraction
acétate
d'éthyle ont
été présentés

ND

de

Méthode de
diffusion sur
disques (AAB).
Technique de
dilution en
milieu liquide
(CMI).
Culture de 0,1
mL à partir de
chaque tube ne
présentant
aucune
croissance
bactérienne sur
des boîtes de
Petri contenant
de gélose
nutritive
(CMB).

(CMB)

$\begin{array}{ll}\text { Dosage colori- } & \text { Le profil des } \\ \text { métrique rapide } & \text { composés } \\ \text { avec le chlorure } & \text { phénoliques des } \\ \text { de p- } & \text { extraits a été } \\ \text { iodonitrotétrazol } & \text { obtenu grâce à CL } \\ \text { ium (CMI) } & \text { avec DMD } \\ & \text { couplée à une IE } \\ & \text { en tandem avec } \\ & \text { SM. Ainsi, 9 } \\ & \text { composés ont été } \\ & \text { identifiés : (Epi) } \\ & \text { catéchine dimer } \\ & \text { type B, (+)- } \\ & \text { catéchine, (-)-Epi } \\ & \text { catéchine, } \\ & \text { Myricétine-O- } \\ & \text { pentoxyl-O- } \\ & \text { hexoside, } \\ & \text { Myricétine-3-O } \\ & \text { rutinoside, } \\ & \text { Myricétine-3-O } \\ \text { glucoside, } & \text { Myricétine-3-O } \\ \text { rhamnoside, } & \text { Quercétine-3-O } \\ \text { rhamnoside et } \\ \text { Kaempferol-3-O } \\ \text { rhamnoside. }\end{array}$

Les principaux composants étudiés par la CPG-FID et CPGSM sont les diterpènes $(87,4 \%)$ dont le composant majeur présentant $31,6 \%$ est le transferruginol. Les principaux composés de l'HE analysés par CPGSM sont : le 3tert-butyl-4méthoxyphénol $(35,02 \%)$, le thymol $(22,83 \%)$ et le cédrénol $(13,60 \%)$. 


\begin{tabular}{|c|c|c|c|c|c|c|c|}
\hline $\begin{array}{l}\text { Ain-Dalfa } \\
\text { localisée à } \\
200 \mathrm{~km} \text { à } \\
\text { l'ouest } \\
\text { d'Alger } \\
\text { (Algérie) } \\
\text { (Djouahri et } \\
\text { al., 2014 b) }\end{array}$ & Cônes & $\begin{array}{l}\text { L'HE a été } \\
\text { extraite par } \\
\text { hydro- } \\
\text { distillation. }\end{array}$ & $\begin{array}{l}\text { Salmonella enterica } \\
\text { E32 (DAI = } 13,5 \pm 0,5 \\
\text { mm) } \\
\text { Klebsiella pneumoniae } \\
\text { CIP } 8291 \text { (DAI }=10,5 \pm \\
0,29 \mathrm{~mm}) \\
\text { Listeria monocytogenes } \\
\text { CIP } 82110 \text { (DAI }=14,5 \\
\pm 0,5 \mathrm{~mm}) \\
\text { Staphylococcus aureus } \\
\text { CIP } 7625 \text { (DAI }=17,5 \pm \\
0,5 \text { mm) } \\
\text { Pseudomonas } \\
\text { aeruginosa CIPA } 22 \\
\text { (DAI }=12,5 \pm 0,5 \text { mm) } \\
\text { Escherichia coli ATTC } \\
10536 \text { (DAI = } 11,5 \pm \\
0,16 \text { mm) }\end{array}$ & $\begin{array}{l}60 \mu \mathrm{g} / \mathrm{ml} \\
10 \mu \mathrm{g} / \mathrm{ml} \\
5 \mu \mathrm{g} / \mathrm{ml} \\
30 \mu \mathrm{g} / \mathrm{ml} \\
40 \mu \mathrm{g} / \mathrm{ml}\end{array}$ & ND & $\begin{array}{l}\text { Méthode de } \\
\text { diffusion sur } \\
\text { disque de papier } \\
\text { (AAB) } \\
\text { Technique de } \\
\text { micro-dilution } \\
\text { en milieu } \\
\text { liquide (CMI) }\end{array}$ & $\begin{array}{l}\text { L'analyse de l'HE } \\
\text { par CPG, } \\
\text { CPG/SM et } \\
\text { HPLC a montré } \\
\text { qu'elle riche en } \\
\text { monoterpènes } \\
\text { oxygénés } \\
\text { (94,47\%) et } \\
\text { pauvre en } \\
\text { hydrocarbures } \\
\text { monoterpènes } \\
(5,53 \%) \text {. Les } \\
\text { composants } \\
\text { majeurs sont } 1 \text { ' } \alpha \\
\text { campholenal } \\
(16,34 \%) \text { le } \\
\text { trans-pinocarveol } \\
(15,45 \%) \text { le cis- } \\
\text { verbénol } \\
(12,36 \%) \text { et le } \\
\text { verbénone } \\
(13,36 \%) \text {. }\end{array}$ \\
\hline $\begin{array}{l}\text { Jardin } \\
\text { botanique } \\
\text { El-Orman à } \\
\text { Giza } \\
\text { (Égypte) } \\
\text { (Ibrahim et } \\
\text { al., 2017) }\end{array}$ & $\begin{array}{l}\text { Partie } \\
\text { aérienne }\end{array}$ & $\begin{array}{l}\text { L'HE a été } \\
\text { extraite par } \\
\text { hydro- } \\
\text { distillation. }\end{array}$ & $\begin{array}{l}\text { Staphylococcus aureus. } \\
\text { Staphylococcus } \\
\text { epidermidis } \\
\text { E. coli } \\
\text { Klebsiella pneumonia } \\
\text { Proteus vulgaris } \\
\text { Pseudomonas } \\
\text { aeruginosa } \\
\text { Shigella boydii }\end{array}$ & $\begin{array}{l}913,1 \mu \mathrm{L} / \mathrm{mL} \\
320 \mu \mathrm{L} / \mathrm{mL} \\
956 \mu \mathrm{L} / \mathrm{mL} \\
828,2 \mu \mathrm{L} / \mathrm{mL} \\
130,3 \mu \mathrm{L} / \mathrm{mL} \\
873,2 \mu \mathrm{L} / \mathrm{mL} \\
757 \mu \mathrm{L} / \mathrm{mL}\end{array}$ & $\begin{array}{l}130,3 \\
\mu \mathrm{L} / \mathrm{mL} \\
551 \\
\mu \mathrm{L} / \mathrm{mL} \\
515,1 \\
\mu \mathrm{L} / \mathrm{mL} \\
94,4 \\
\mu \mathrm{L} / \mathrm{mL} \\
95,4 \\
\mu \mathrm{L} / \mathrm{mL} \\
27,6 \\
\mu \mathrm{L} / \mathrm{mL} \\
460 \\
\mu \mathrm{L} / \mathrm{mL}\end{array}$ & $\begin{array}{l}\text { Méthode de la } \\
\text { micro-dilution. }\end{array}$ & $\begin{array}{l}\text { Les principaux } \\
\text { composants } \\
\text { analysés par } \\
\text { CPG/SM sont : le } \\
\text { camphre } \\
(21,23 \%) \text {, } \\
\text { l'acétate de } \\
\text { bornyle }(15,03 \%) \text {, } \\
\text { le }(+)-\text { fenchol } \\
(13,85 \%), \text { le } \\
\text { fenchone }(9,48 \%) \\
\text { et l'acétate } \\
\text { d'isobornyle } \\
(8,39 \%) .\end{array}$ \\
\hline
\end{tabular}

AAB : activité antibactérienne ; BLSE : bêta lactamases à spectre élargi ; CL : chromatographie en phase liquide ; CMB : concentration minimale bactéricide correspondant à la plus faible concentration de l'extrait ou de l'huile essentielle pour laquelle le microorganisme incubé est totalement tué ; CMI : concentration minimale inhibitrice représentant la plus faible concentration de l'extrait ou de l'huile essentielle pour laquelle la croissance de microorganisme est totalement inhibée ; CPG/SM : chromatographie en phase gazeuse couplée à la spectrométrie de masse ; DAI : diamètre d'auréole d'inhibition; DMD : détection à matrice de diodes; $\boldsymbol{E}$. coli : Escherichia coli; H : Hammam Melouane; HE : huile essentielle ; HEC : huile essentielle des cônes ; HEF : huile essentielle des feuilles; IE : ionisation électrospray; MHA : milieu Mueller Hinton; ND : non déterminé ; SARM : Staphylococcus aureus résistant à la méthicilline; SASM : Staphylococcus aureus sensible à la méthicilline; $\mathbf{T}$ : Tipaza.

\subsubsection{Activité antifongique}

Les phytopathologies causées par les microorganismes, notamment les champignons, sont un obstacle majeur qui touche de nombreuses cultures agricoles, menant ainsi à leur altération et à de grandes pertes économiques (Zahir, 2016 ; Zahir et al., 2018 b). Actuellement, les plantes infestées sont traitées à l'aide de pesticides. Cependant, cette méthode conventionnelle pollue l'environnement et présente un effet néfaste pour la santé humaine (Zahir et al., 2018 b). Pour faire face à cela, les plantes médicinales sont utilisées dans le cadre du contrôle biologique. 
En effet, elles présentent des bio-ressources très riches en principes actifs (Zahir, 2016). C'est dans ce contexte que l'activité antifongique de l'HE des branches terminales ligneuses et des racines de $T$. articulata de Tunisie a été étudiée et que la composition chimique des fractions volatiles a été identifiée par CPG couplée à un détecteur à ionisation de flamme (CPG-FID) et par la CPG-SM. Les résultats montrent que les branches terminales ligneuses présentent vingt composés constituant $83,7 \%$ de l'HE contre 29,2\% pour les composés issus des racines. L'étude montre aussi que le nonan-1-ol est le constituant principal (75,22\%). Les hydrocarbures monoterpéniques, à savoir le terpinène $(3,04 \%)$, le $\Delta-3$ carène $(1,17 \%)$ et l'ocimène $(1,09 \%)$ sont les principaux composés. Pour l'HE des racines, les principaux composés détectés sont l'acétate de bornyle $(16,63 \%)$, le camphène $(1,59 \%)$ et le cadinène $(2,86 \%)$. Cette partie est principalement riche en composés oxygénés $(20,25 \%)$ et en hydrocarbures sesquiterpéniques (6,84\%). Par la suite, les HE ont été testées pour leur activité antifongique contre cinq champignons phytopathogènes, à savoir: Fusarium solani cucurbitae, Alternaria solani, F. oxysporum niveum, Botrytis cinerea et Rhizoctonia solani. Les résultats des essais biologiques contre $F$. solani cucurbitae $(11,3 \pm 0,6 \mathrm{~mm})$ et $F$. oxysporum $f$. sp. niveum $(16,6 \pm 1,7 \mathrm{~mm})$ pourraient être liés au fort pourcentage d'alcools aliphatiques, en particulier le nonan-1-ol.

Il ressort de cette étude que les composés volatils des branches terminales ligneuses et des racines de $T$. articulata pourraient être une source prometteuse pour un traitement antimicrobien à utiliser comme pesticide non conventionnel pour le contrôle des maladies des plantes (Tekaya-karoui et al., 2011). Notons que l'activité antifongique des extraits et/ou les HE de T. articulata a été évaluée davantage par d'autres recherches (tableau 2).

Tableau 2 : Activité antifongique de Tetraclinis articulata

\begin{tabular}{|c|c|c|c|c|c|c|c|}
\hline Région & $\begin{array}{l}\text { Partie } \\
\text { utilisée }\end{array}$ & Extrait/ HE * & $\begin{array}{c}\text { Activité contre les } \\
\text { bactérie (s) sensible (s) }\end{array}$ & CMI & CMF & $\begin{array}{c}\text { Méthode (s) } \\
\text { utilisée (s) }\end{array}$ & $\begin{array}{l}\text { Composé (s) } \\
\text { majeur (s) ou } \\
\text { actif (s) }\end{array}$ \\
\hline $\begin{array}{l}\text { Khémisset } \\
\text { (Maroc) } \\
\text { (Bourkhiss } \\
\text { et } a l ., \text { 2007) }\end{array}$ & Feuilles & $\begin{array}{l}\text { L'HE a été } \\
\text { extraite par } \\
\text { hydro- } \\
\text { distillation }\end{array}$ & $\begin{array}{l}\text { Penicellium parasiticus } \\
\text { Aspergillus niger }\end{array}$ & $\begin{array}{l}1 / 500 \mathrm{~V} / \mathrm{V} \\
1 / 500 \mathrm{~V} / \mathrm{V}\end{array}$ & ND & $\begin{array}{l}\text { Différentes } \\
\text { concentrations } \\
\text { de l'HE ont été } \\
\text { incorporées } \\
\text { dans le milieu } \\
\text { PDA agar. }\end{array}$ & $\begin{array}{l}\text { Les principaux } \\
\text { composés de l'H.E } \\
\text { ont été identifiés } \\
\text { par CPG et } \\
\text { CPG/SM: l'acétate } \\
\text { de bornyle }(30,74 \\
\%), 1 \text { ' } \alpha \text {-pinène } \\
(23,54 \%) \text {, le } \\
\text { camphre }(17,27 \\
\%) \text { et le limonène } \\
(23,31 \%) \text {. }\end{array}$ \\
\hline
\end{tabular}


Bulletin de la Société Royale des Sciences de Liège, Vol. 89, articles, 2020, p. 91 - 114

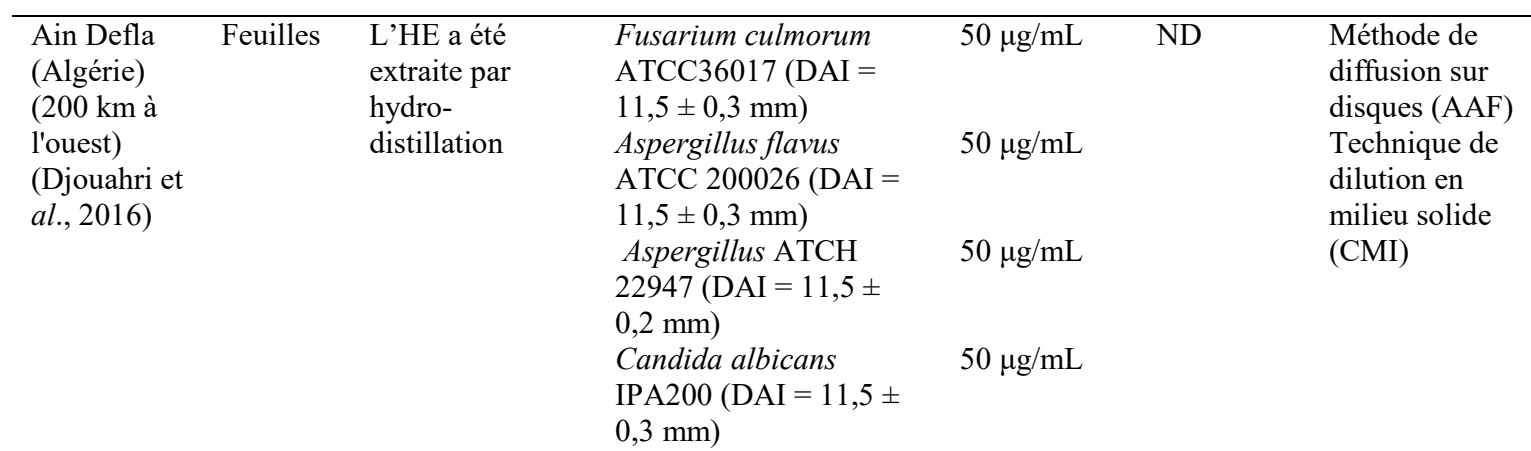

\begin{tabular}{|c|c|c|c|}
\hline $\begin{array}{l}\text { Korbous } \\
\text { (Nabeul, } \\
\text { Tunisie) } \\
\text { (Ben } \\
\text { Ghnaya et } \\
\text { al., 2016) }\end{array}$ & Feuilles & $\begin{array}{l}\text { L'HE a été } \\
\text { extraite par } \\
\text { hydro- } \\
\text { distillation }\end{array}$ & $\begin{array}{l}\text { Les pourcentages } \\
\text { d'inhibition de la } \\
\text { croissance des hyphes } \\
\text { sont d'ordre : } 71,17 \pm \\
5,41 \% ; 56,89 \pm \\
3,33 \% ; 46,42 \pm 5,05 \% ; \\
29,82 \pm 4,96 \% ; 25,36 \pm \\
6,16 \% \text { contre Botrytis } \\
\text { cinerea ; Fusarium } \\
\text { avenaceum, Fusarium } \\
\text { oxysporum, Fusarium } \\
\text { culmorum, Fusarium } \\
\text { solani, respectivement. }\end{array}$ \\
\hline
\end{tabular}

ND ND $\quad$ L'HE a été
incorporée dans
le milieu PDA
agar pour
déterminer
l'inhibition de
la croissance
des hyphes en
utilisant le test
de contact in
vitro (AAF).

\begin{tabular}{lllllll}
\hline Jardin & Partie & L'HE a été & Candida albicans & $0,659 \pm$ & $1,515 \pm$ & Méthode de la \\
botanique & aérienne & extraite par & & 0,053 & 0,085 & microdilution. \\
El-Orman à & & hydro- & & $\mu \mathrm{L} / \mathrm{mL}$ & $\mu \mathrm{L} / \mathrm{mL}$ & \\
Giza & & distillation & Candida glabrata & $<64 \mu \mathrm{L} / \mathrm{mL}$ & $\mathrm{ND}$ & \\
(Égypte) & & & Candida krusei & $<64 \mu \mathrm{L} / \mathrm{mL}$ & $\mathrm{ND}$ & \\
(Ibrahim et & & Candida parapsilosis & $0,659 \pm$ & $1,148 \pm$ & \\
al., 2017) & & & 0,042 & 0,087 & \\
& & & $\mu \mathrm{L} / \mathrm{mL}$ & $\mu \mathrm{L} / \mathrm{mL}$
\end{tabular}

Les composants par CPG et CPG-

SM sont : l' $\alpha$ pinène $(29,0 \%)$,

l'acétate de bornyle $(25,5 \%)$,

le camphre $(16,6 \%)$ et le limonène $(6,7 \%)$ représentant $96,6 \%$ du total de l'HE. Les composés ont été identifiés par CPG et CPG-SM. Ils représentent 91\% de la composition de l'HE avec une majorité d'hydrocarbures monoterpènes (62,80\%), en particulier l'épinène $(56,21 \%)$ et le myrcène $(3,08 \%)$. Les principaux composants analysés par CPG/SM sont : le camphre (21,23\%), l'acétate de bornyle $(15,03 \%)$, le $(+)$ - fenchol (13,85\%), le fenchone $(9,48 \%)$ et l'acétate d'isobornyle $(8,39 \%)$.

\begin{tabular}{llll}
\hline $\begin{array}{l}\text { Jardin } \\
\text { botanique }\end{array}$ & $\begin{array}{l}\text { Feuille } \\
\text { et tige }\end{array}$ & $\begin{array}{l}\text { Extrait } \\
\text { méthanolique }\end{array}$ & $\begin{array}{l}\text { L'extrait méthanolique } \\
\text { réduit } 55,8 \% \text { et } 40,3 \% \\
\text { de la croissance }\end{array}$ \\
Alger & & & mycélienne de \\
(Algérie) & & & Fusarium culmorum et \\
$\left(36^{\circ} 43\right.$ '15.3 & & & de $F$. graminearum, in \\
'N & & & vitro, respectivement.
\end{tabular}

$3^{\circ} 09^{\prime} 02.4^{\prime \prime}$

E)

(Dane et

al., 2015)

$\begin{array}{ll}\text { ND ND } & \text { L'extrait } \\ & \text { méthanolique a } \\ & \text { été incorporé } \\ & \text { dans le milieu } \\ & \text { PDA pour } \\ & \text { déterminer } \\ & \text { l'inhibition de } \\ & \text { la croissance de } \\ & \text { mycélienne. }\end{array}$

Les principaux

composés analysés par UPLC couplée à la $\mathrm{PDA}$ et $\mathrm{SM}$ sont les composés phénoliques : myricétinerhamnoside $(42,76 \%)$ quercétine -3-Orhamnoside (31,05\%), catéchine $(18,83 \%)$ et myricétine-hexose $(4,58 \%)$

\begin{tabular}{|c|c|c|c|c|c|}
\hline $\begin{array}{l}\text { Khemisset } \\
\text { (Maroc) } \\
\text { (Fidah et } \\
\text { al., 2019) }\end{array}$ & $\begin{array}{l}\text { Loupe } \\
\text { de } \\
\text { racine }\end{array}$ & $\begin{array}{l}\text { L'HE a été } \\
\text { extraite par } \\
\text { hydro- } \\
\text { distillation }\end{array}$ & $\begin{array}{l}\text { Gloeophyllum trabeum } \\
\text { Oligoporus placenta } \\
\text { Coniophora puteana } \\
\text { Trametes versicolor }\end{array}$ & $\begin{array}{l}1 / 5000(\mathrm{v} / \mathrm{v}) \\
1 / 5000(\mathrm{v} / \mathrm{v}) \\
1 / 4000(\mathrm{v} / \mathrm{v}) \\
1 / 4000(\mathrm{v} / \mathrm{v})\end{array}$ & $\begin{array}{l}\text { Technique de } \\
\text { contact direct } \\
\text { sur milieu } \\
\text { gélose }\end{array}$ \\
\hline
\end{tabular}
Les composants majeurs analysés par la CPG-FID et la CPG-SM sont : le thymol, 3-tertbutyl-4methoxyphénol, le cédrol et l' $\alpha$ cédrène 
AAF : activité antifongique ; CPG-FID : chromatographie en phase gazeuse couplée à un détecteur à ionisation de flamme ; E : Est ; ENSA : Ecole Nationale Supérieure Agronomique ; F : Fusarium; HPLC : chromatographie en phase liquide à haute performance; $\mathbf{N}$ : nord; Milieu PDA : gélose à la pomme de terre et au dextrose; PDA : détecteur à réseau de photodiodes ; UPLC : chromatographie liquide ultra-performante.

Les propriétés antimicrobiennes sont liées éventuellement aux métabolites de la plante, y compris les terpènes, les flavonoïdes et les polyphénols (Talbaoui et al., 2016). Plusieurs recherches ont démontré que ces molécules possèdent également un effet antimicrobien élevé (Tiwari et Husain, 2017 ; Talbaoui et al., 2016 ; Chraibi et al., 2020). On citera, à titre d'exemple, l'a-pinène, le camphre, le bornéol, l'acétate de bornyle et le thymol, monoterpènes connus par leur fort potentiel d'activité antimicrobienne (Bourkhiss et al., 2007 ; El Moussaouiti et al., 2010 ; Chikhoune et al., 2013 ; Akbli et al., 2016 ; Chraibi et al., 2020 ; Zahir et Rahmani, 2020). Le mode d'action de ces composés est dû notamment à leur capacité à perturber les membranes, provoquant ainsi la lyse cellulaire (Tiwari et Husain, 2017 ; Talbaoui et al., 2016). Fidah et al., (2019) ont également rapporté une activité inhibitrice de composés phénoliques sur des enzymes fongiques dont le site actif contient des fonctions thiol (-SH). Quant aux flavonoïdes, qui ont la capacité d'inactiver les adhésines microbiennes, les enzymes et les protéines de transport cellulaire (Tiwari et Husain, 2017), ils présentent un effet inhibiteur sur le métabolisme énergétique et la synthèse des acides nucléiques (Rached et al., 2018).

\subsubsection{Activité antioxydante}

Les radicaux libres se forment constamment dans le corps humain comme produits de métabolisme normal (Ben Jemia et al., 2013 ; Sayah et al., 2017). Ils sont impliqués dans diverses situations pathologiques induisant les maladies cardiovasculaires, l'athérosclérose, la polyarthrite rhumatoïde, le cancer, les troubles neurologiques (Parkinson, Alzheimer), le diabète et le vieillissement (Valko et al., 2007 ; El Jemli et al., 2016 b). Ces radicaux libres instables peuvent être éliminés par des antioxydants qui diminuent le taux d'oxydation et protègent les cellules des dommages (El-Omari et al., 2019). Les antioxydants sont également ajoutés dans les aliments pour prévenir ou retarder l'auto-oxydation lipidique, phénomène radicalaire bien connu et favorisé par oxygène de l'air, la lumière et la température (Mamta et al., 2012). Cependant, malgré l'efficacité des antioxydants synthétiques, leur utilisation dans les industries agroalimentaire, cosmétique et pharmaceutique est associée à des effets néfastes pour la santé (Bourkhiss et al., 2010 ; Sayah et al., 2017). En effet, des réactions secondaires 
négatives de l'hydroxyanisole butylé (BHA) et de l'hydroxytoluène butylé (BHT) ont été documentées (Ben Jemia et al., 2013). Des études montrent que le BHT a une action sur la thyroïde du rat avec des modifications hormonales de la concentration des hormones thyroïdiennes, une hyperplasie et des tumeurs de la thyroïde (ANSES, 2016). Compte tenu de ces propriétés, il est primordial de chercher d'autres alternatives comme celles de l'utilisation d'extraits et/ou des HE de plantes médicinales en tant que sources potentielles d'antioxydants avec des effets secondaires limités ou inexistants (Sayah et al., 2017). Incontestablement, l'intérêt pour les plantes, sources naturelles d'antioxydants, croît avec leur utilisation pour la prévention de l'oxydation des aliments et pour le traitement thérapeutique de plusieurs maladies causées par le stress oxydatif (Ben Jemia et al., 2013 ; Mamta et al., 2012). C'est dans ce contexte qu'a été mise en évidence, in vitro, l'activité antioxydante de l'HE et de l'extrait à l'acétone et l'eau (80:20, v/v) des feuilles de $T$. articulata de la région de Bou Karnine (Tunisie). L'analyse par CPG/SM de l'HE a permis d'identifier 66 composants qui constituent 93,5\% de l'huile. Les principaux constituants sont l'acétate de bornyle $(31,4 \%)$, l' $\alpha$-pinène $(24,5 \%)$ et le camphre (20,3\%). Les activités antioxydantes des échantillons ont été déterminées à l'aide de quatre méthodes différentes, à savoir : 2,2-diphényl-1picrylhydrazyle (DPPH), $\beta$-carotène/acide linoléique, pouvoir réducteur et activité chélatante des métaux. Les résultats des tests du système DPPH ont montré que l'activité antioxydante la plus puissante a été produite par l'extrait préparé (concentration inhibitrice médiane $\mathrm{CI}_{50}$ égale à $5,5 \mathrm{mg} / \mathrm{mL}$ ) et que cette activité est deux fois plus élevée que celle du BHT utilisé comme contrôle positif $\left(\mathrm{CI}_{50}\right.$ égale à $\left.12 \mathrm{mg} / \mathrm{mL}\right)$. La quantité totale de composés phénoliques, flavonoïdes et de tanins est aussi très élevée dans l'extrait. De plus, il y a une forte corrélation entre le potentiel d'activité antioxydante et la concentration en phénols totaux de l'extrait (Ben Jemia et al., 2013).

Par ailleurs, dans une autre étude effectuée sur deux échantillons de feuilles et de cônes de $T$. articulata provenant de deux sites différents en Algérie (Hammam Melouane et Tipaza), l'activité antioxydante des HE a été testée par l'utilisation du procédé de piégeage du radical libre DPPH. En comparaison avec le BHT et le BHA, utilisés comme témoins positifs, tous les échantillons ont montré une faible activité (Chikhoune et al., 2013).

D'autres études encore ont démontré des résultats surprenants concernant l'activité antioxydante de la plante (tableau 3). 
Tableau 3 : Activité antioxydante de Tetraclinis articulata

\begin{tabular}{|c|c|c|c|c|c|}
\hline Région & Partie utilisée & Extrait/ HE & Modèle expérimental & Effet ou résultat & Principes actifs \\
\hline $\begin{array}{l}\text { Marrakech } \\
\text { Ait Aissi } \\
\text { Ihahane } \\
(30910 \mathrm{~N} \text { et } \\
09430 \mathrm{O}) \\
\text { (El Jemli et } \\
\text { al., 2016 a) }\end{array}$ & Feuilles & $\begin{array}{l}\text { L'HE a été extraite par } \\
\text { hydro-distillation }\end{array}$ & $\begin{array}{l}\text { Le potentiel antioxydant } \\
\text { a été étudié à l'aide des } \\
\text { méthodes suivantes : la } \\
\text { capacité de neutraliser } \\
\text { DPPH, la capacité } \\
\text { d'inhiber l'ABTS et le } \\
\text { dosage du pouvoir } \\
\text { antioxydant de la } \\
\text { réduction du fer } \\
\text { (FRAP). }\end{array}$ & $\begin{array}{l}\text { Les valeurs } \mathrm{CI}_{50} \text { sont } \\
\text { de l'ordre } 12,05 \pm \\
0,24 \mathrm{mg} / \mathrm{mL}, 8,90 \pm \\
0,17 \mathrm{mg} / \mathrm{mL} \text { et } 0,15 \\
\pm 0,01 \mathrm{mg} / \mathrm{mL} \text {, } \\
\text { respectivement }\end{array}$ & $\begin{array}{l}\text { Les principaux } \\
\text { composés identifiés } \\
\text { dans l'analyse CPG / } \\
\text { SM sont l'acétate de } \\
\text { bornyle }(26,81 \%), \text { le } \\
\text { camphre }(22,40 \%) \text { et } \\
\text { l' } \alpha \text {-pinène }(7,16 \%) \text {, } \\
\text { avec } 25 \text { autres } \\
\text { constituants mineurs. }\end{array}$ \\
\hline $\begin{array}{l}\text { Ain Defla } \\
\text { (Algérie) } \\
(200 \mathrm{~km} \text { à } \\
\text { l'ouest) } \\
\text { (Djouahri et } \\
\text { al., 2016) }\end{array}$ & Feuilles & $\begin{array}{l}\text { L'HE a été extraite par } \\
\text { hydro-distillation }\end{array}$ & $\begin{array}{l}\text { L'activité antioxydante } \\
\text { a été évaluée en } \\
\text { utilisant les radicaux } \\
\text { libres de DPPH et le } \\
\text { dosage du pouvoir } \\
\text { antioxydant réducteur } \\
\text { de fer (FRAP). }\end{array}$ & $\begin{array}{l}\text { Les valeurs diffèrent } \\
\text { selon la méthode } \\
\text { utilisée : } \\
\text { DPPH : } \mathrm{CI}_{50}=517,6 \\
\pm 1,75 \mu \mathrm{g} / \mathrm{mL} . \\
\mathrm{FRAP}: \mathrm{CE}_{50}=38,2 \\
\pm 0,29 \mu \mathrm{g} / \mathrm{mL} . \\
\text { Cependant, ces } \\
\text { valeurs sont plus } \\
\text { faibles que celles } \\
\text { obtenues par BHA et } \\
\text { BHT utilisés comme } \\
\text { composés de } \\
\text { référence. }\end{array}$ & $\begin{array}{l}\text { Les composants } \\
\text { majeurs analysés par } \\
\text { CPG et CPG-SM } \\
\text { sont : l' } \alpha \text {-pinène } \\
(29,0 \%), \text { l'acétate de } \\
\text { bornyle }(25,5 \%), \text { le } \\
\text { camphre }(16,6 \%) \text { et le } \\
\text { limonène }(6,7 \%) \\
\text { représentant } 96,6 \% \text { du } \\
\text { total de l'HE. }\end{array}$ \\
\hline $\begin{array}{l}\text { Zeddine, } \\
\text { Mansoura, } \\
\text { Zakkar, } \\
\text { Tazoult } \\
\text { (Algérie) } \\
\text { (Djouahri et } \\
\text { al., 2014 a) }\end{array}$ & $\begin{array}{l}\text { Feuilles } \\
\text { Bois }\end{array}$ & $\begin{array}{l}\text { L'HE a été extraite par } \\
\text { hydro-distillation }\end{array}$ & $\begin{array}{l}\text { Le potentiel antioxydant } \\
\text { a été étudié à l'aide de la } \\
\text { méthode : capacité de } \\
\text { neutraliser DPPH en } \\
\text { utilisant BHA et BHT } \\
\text { comme référence. }\end{array}$ & $\begin{array}{l}\text { Les valeurs } \mathrm{CI}_{50} \\
\text { déterminées par le } \\
\text { DPPH varient de } \\
88,44 \pm 3,27 \text { à } 119,44 \\
\pm 5,25 \mathrm{~g} / \mathrm{mL} \text { et de } \\
113,47 \pm 4,19 \text { à } \mathrm{g} / \mathrm{mL} \\
\text { pour le bois et les } \\
\text { feuilles, } \\
\text { respectivement. }\end{array}$ & $\begin{array}{l}\text { Les composants } \\
\text { majeurs ont été } \\
\text { analysés par CPG et } \\
\text { CPG-SM. Ainsi, } 34 \\
\text { composés ont été } \\
\text { identifiés, représentant } \\
\text { 90,6-97,6\% de l'HE } \\
\text { des feuilles. Les } \\
\text { composés } \\
\text { prédominants sont : } \\
\text { l'épinène (16,3- } \\
25,3 \%), \text { le camphre } \\
(17,2-21,4 \%) \text { et } \\
\text { l'acétate de bornyle. } \\
(17,6-20,6 \%) . \\
29 \text { composés ont été } \\
\text { identifiés, représentant } \\
91,2 \text { à } 95,5 \% \text { de } \\
\text { l'huile de bois totale. } \\
\text { Le camphène (15,5 à } \\
18,8 \%), \text { le cédrol } \\
(10,5 \text { à } 12,7 \%) \text { et } \\
\text { l'acorénol }(8,2 \text { à } \\
10,4 \%) \text { sont les } \\
\text { composés majeurs. }\end{array}$ \\
\hline $\begin{array}{l}\text { Aït Issli } \\
\text { Ihahan } \\
\left(\mathrm{N} 30^{\circ} 91\right. \\
\text { /O } 09^{\circ} 43 \text { ) } \\
\text { (Maroc) } \\
\text { (El Jemli et } \\
\text { al., 2016 b) }\end{array}$ & Feuilles & Extrait aqueux (E) & $\begin{array}{l}\text { L'activité antioxydante } \\
\text { a été évaluée par la } \\
\text { capacité de piégeage } \\
\text { des radicaux de DPPH, } \\
\text { la capacité d'inhiber } \\
\text { l'ABTS et le dosage du } \\
\text { pouvoir antioxydant de } \\
\text { la réduction du fer } \\
\text { (FRAP). }\end{array}$ & $\begin{array}{l}\text { Les valeurs } \mathrm{CI}_{50} \text { sont } \\
\text { de l'ordre de : } \\
27,38 \pm 0,02 \mu \mathrm{g} / \mathrm{ml} \text {, } \\
32,92 \pm 0,56 \mu \mathrm{g} / \mathrm{ml} \text { et } \\
47,12 \pm 0,15 \mu \mathrm{g} / \mathrm{ml} \text {, } \\
\text { respectivement. }\end{array}$ & $\begin{array}{l}\text { La teneur totale en } \\
\text { polyphénols dans l'E } \\
\text { en utilisant la méthode } \\
\text { de Folin Ciocalteu est } \\
175,67 \pm 10,21 \mu \mathrm{g} \\
\text { EAG/mg de l'E de } \\
\text { plante, alors que la } \\
\text { teneur des flavonoïdes } \\
\text { en employant la } \\
\text { méthode d'AlCl }{ }_{3} \text { est } \\
11,78 \pm 0,30 \mu \mathrm{g} \\
\mathrm{EQ} / \mathrm{mg} \text { de l'E de } \\
\text { plante. }\end{array}$ \\
\hline
\end{tabular}




\begin{tabular}{llll}
\hline Khemisset & Feuilles, & L'HE a été extraite par & Les propriétés anti \\
(Maroc) & Rameaux & hydro-distillation & oxydantes ont été \\
(Bourkhiss & Sciure de bois & & évaluées par trois tests : \\
et al., 2010) & de tronc & & le test de piégeage du \\
& & & radical DPPH, le test de \\
& & décoloration du $\beta-$ \\
& & carotène et le pouvoir \\
& & réducteur (FRAP).
\end{tabular}

Les HE du tronc et
des feuilles
manifestent un
important potentiel
antioxydant, avec les
trois méthodes,
supérieur à celui du
composé antioxydant
de référence (BHT) et
des rameaux,
respectivement.

Les composants majeurs ont été analysés par la CGP et la CGP-SM. Ainsi, des feuilles et des rameaux sont principalement constituées de monoterpènes hydrocarbonés (respectivement $30,8 \%$ et $55,53 \%$ ) et oxygénés (respectivement $60,9 \%$ et $10,16 \%$ ). Les rameaux sont relativement plus riches en sesquiterpènes $(15,08 \%)$.

\begin{tabular}{|c|c|c|c|c|}
\hline $\begin{array}{l}\text { Korbos } \\
\text { (situé dans } \\
\text { la région du } \\
\text { Cap Bon à }\end{array}$ & Feuilles & $\begin{array}{l}\text { L'HE a été extraite par } \\
\text { hydro-distillation. }\end{array}$ & $\begin{array}{l}\text { L'activité antioxydante } \\
\text { a été mesurée selon } \\
\text { deux méthodes : ABTS } \\
\text { et DPPH. }\end{array}$ & $\begin{array}{l}\text { Les valeurs } \mathrm{CI}_{50} \text { sont } \\
\text { de } 324 \pm 14 \mathrm{mg} / \mathrm{L} \text { et } \\
3681 \pm 69 \mathrm{mg} / \mathrm{L} \text {, } \\
\text { respectivement }\end{array}$ \\
\hline
\end{tabular}

$60 \mathrm{~km}$ )

Tunisie (Herzi et al., 2013)

\begin{tabular}{lll}
\hline Mornag et & Résine & ND \\
Gouvernorat & & $\begin{array}{l}\text { Le potentiel antioxydant } \\
\text { a été étudié de la résine } \\
\text { de Ben }\end{array}$ \\
arous & à l'aide de cinq tests à \\
savoir : DPPH, ABTS, \\
(Nord-Est & pouvoir réducteur, la \\
de la & catalase et de la \\
Tunisie) & paraoxonase. \\
(Jlizi et $a l .$, &
\end{tabular}

(Jlizi et al. 2018)

\begin{tabular}{lll}
\hline Oran & Feuilles & Une extraction par \\
(Ouest de & & partage liquide-liquide \\
l'Algérie) & a été effectuée pour \\
(Rached et & obtenir les fractions \\
al., 2018) & organiques de l'extrait \\
& aqueux en utilisant de \\
& l'acétate d'éthyle et du \\
& butanol, \\
& successivement.
\end{tabular}

L'activité anti-oxydante de la fraction d'acétate d'éthyle à $10 \mathrm{mg} / \mathrm{mL}$ a été mesurée selon méthodes : DPPH, FRAP, le test de décoloration du $\beta$ carotène et inhibition de la peroxydation lipidique dans les homogénats de cerveau de porc à l'aide du dosage des substances réactives à l'acide thiobarbiturique (TBARS)

Les composants
majeurs analysés par la CPG-FID et la CPG-SM sont : l' $\alpha$ pinène $(24,9 \%)$, l'acétate de linalol $(21,44 \%)$ et l'oxyde de caryophyllène (4,24\%).

Les valeurs diffèrent selon la méthode utilisée :

$\mathrm{DPPH}: \mathrm{CI}_{50}=59 \pm 2$

$\mu \mathrm{g} / \mathrm{mL}$.

ABTS : $\mathrm{CI}_{50}=76 \pm 2$

$\mu \mathrm{g} / \mathrm{mL}$

FRAP : $\mathrm{CI}_{50}=189 \pm$

$3 \mu \mathrm{g} / \mathrm{mL}$.

Catalase : $399.2 \pm 1.0$

$\mu \mathrm{mol}$ de $\mathrm{H}_{2} \mathrm{O}_{2}$

détruite par min par

$\mathrm{mg}$ de protéine.

Paraoxonase : $27.0 \pm$

$1 \mu \mathrm{M} / \mathrm{min} / \mathrm{L}$.

Les valeurs diffèrent Le profil des

selon la méthode composés phénoliques

utilisée :

DPPH : $\mathrm{CE}_{50}=4,5 \pm$

$0,2 \mu \mathrm{g} / \mathrm{mL}$.

FRAP : $\mathrm{CE}_{50}=3,84 \pm$

$0,01 \mu \mathrm{g} / \mathrm{mL}$.

Décoloration du $\beta-$

carotène : $\mathrm{CE}_{50}=779$

$\pm 92 \mu \mathrm{g} / \mathrm{mL}$

Inhibition de la

peroxydation

lipidique : $\mathrm{CE}_{50}=$ $1,76 \pm 0,08 \mu \mathrm{g} / \mathrm{mL}$ principaux par la CPG-FID et

CPG-SM sont les

diterpènes $(87,4 \%)$

dont le composant

majeur présentant

$31,6 \%$ est le

transferruginol. composants étudiés des extraits a été

obtenu grâce à $\mathrm{CL}$

avec DMD couplée à

une IE en tandem avec

SM. Ainsi, 9

composés ont été

identifiés : (Epi) catéchine dimer type $\mathrm{B},(+)$-catéchine, (-)Epicatéchine,

Myricétine-O-

pentoxyl-O-hexoside,

Myricétine-3-O

rutinoside, Myricétin-

3-O glucoside,

Myricétine-3-O

rhamnoside,

Quercétine-3-O

rhamnoside et

Kaempférol-3-O

rhamnoside. 
Bulletin de la Société Royale des Sciences de Liège, Vol. 89, articles, 2020, p. 91 - 114

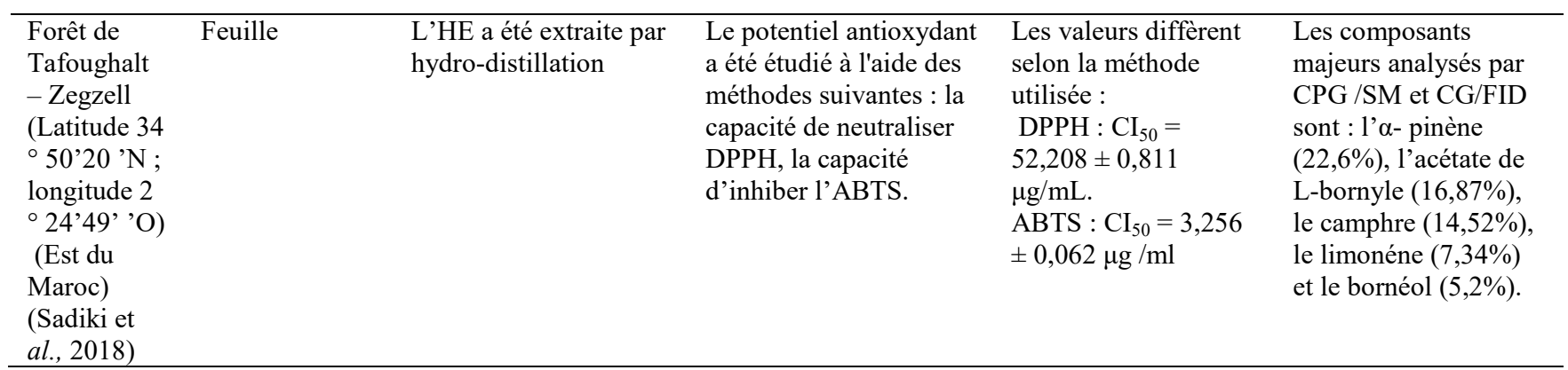

BHT : butylhydroxytoluène; BHA : butylhydroxyanisole; $\mathbf{C E}_{\mathbf{5 0}}$ : concentration fournissant 0,5 d'absorbance a été calculée en traçant l'absorption à $700 \mathrm{~nm}$ en fonction de la concentration correspondante (Djouhari et al., 2016) ou concentration d'extrait correspondant à 50\% de l'activité anti-oxydante (Rached et al., 2018); $\mathbf{C I}_{\mathbf{5 0}}$ : concentration inhibitrice médiane ; DPPH : 2,2-diphényl-1-picrylhydrazyle ; FRAP : pouvoir antioxydant de la réduction du fer; E : extrait ; EAG : équivalents d'acide gallique ; EQ : équivalents de quercétine ; $\mathbf{O}$ : ouest.

D’après le tableau 3, les études phytochimiques opérées sur le thuya de barbarie ont révélé la présence de plusieurs composés, principalement les flavonoïdes et les polyphénols. Ces métabolites sont généralement impliqués dans de nombreuses activités biologiques, essentiellement dans la prévention du stress oxydatif (Sayah et al., 2017). En effet, ils sont considérés comme des antioxydants forts et ils se révèlent plus efficaces que les vitamines $\mathrm{C}$ ou E et les caroténoïdes (Mamta et al., 2012). L’activité réductrice des composés phénoliques et des flavonoïdes dépend du nombre de fonctions hydroxyles libres qui sont présents dans leurs structures chimiques et qui piègent les radicaux libres (Mamta et al., 2012; Tiwari et Husain, 2017). Une telle activité favorise la prévention contre les radicaux libres responsables du stress oxydatif et de plusieurs maladies associées (Tiwari et Husain, 2017). On citera, à titre d'exemple, le cas des catéchines qui semblent être les flavonoïdes les plus puissants pour la protection de l'organisme contre les espèces réactives de l'oxygène. Il a été rapporté également que les dérivés de la quercétine, l'épicatéchine, le kaempférol 3-O- $\alpha$-rhamnoside et la myricétine-3-O-rhamnoside possèdent des activités antioxydantes (Mamta et al., 2012 ; Rached et al., 2018). La présence de tels composés dans les extraits du T. articulata explique l'effet antioxydant de cette essence forestière.

Par ailleurs, le pouvoir antioxydant serait également lié aux monoterpènes, aux diterpènes et aux sesquiterpènes oxygénés (Bourkhiss et al., 2010 ; Jemli et al., 2016 a ; Sadiki et al., 2018 ; Bouyahya et al., 2019 ; Chraibi et al., 2020). Incontestablement, la présence d'apinène, d'acétate de L-bornyle, de camphre et de bornéol (Bourkhiss et al., 2010 ; El Jemli et al., 2016 a ; Ben Jemia et al., 2013 ; Sadiki et al., 2018) dans les extraits aqueux, organiques et/ou dans les HE d'espèces végétales constitue une source d'activités antioxydantes 
importantes. Par conséquent, l'effet antiradicalaire de T. articulata pourrait être dû à l'action d'un seul métabolite ou à une synergie entre plusieurs des métabolites en présence.

\subsubsection{Activité anti-inflammatoire}

L'activité anti-inflammatoire des HE de T. articulata, et plus précisément des feuilles et bois collectés dans quatre sites en Algérie (Zeddine, Mansoura, Zakkar et Tazoult), a été évaluée et que le pourcentage d'inhibition de la lipoxygénase et de la xanthine oxydase a été mis en évidence via la détermination des valeurs de $\mathrm{CI}_{50}$ (inhibition de $50 \%$ de l'activité enzymatique). L'étude des HE a montré qu'elles possèdent des propriétés anti-inflammatoires marquées, bien que les valeurs produites par les deux essais soient quantitativement différentes pour les deux organes. Des différences significatives au niveau de l'activité recherchée ont été observées également dans les différents sites d'étude. Ainsi, les études menées montrent que la lipooxygénase est efficacement inhibée avec une $\mathrm{CI}_{50}$ allant respectivement pour le bois et les feuilles de 2,51 $\pm 0,23$ à 8,88 $\pm 0,11 \mathrm{~g} / \mathrm{mL}$ et de 13,86 \pm 0,2 à 25,93 $\pm 0,16 \mathrm{~g} / \mathrm{ml}$. Les plus fortes inhibitions ont été observées pour des HE de la région de Zeddine, avec des valeurs de $\mathrm{CI}_{50}$ de 2,51 $\pm 0,23 \mathrm{~g} / \mathrm{mL}$ et 13,86 $\pm 0,2 \mathrm{~g} / \mathrm{mL}$, respectivement pour le bois et pour les feuilles, tandis que la plus faible inhibition a été observée pour des HE de Tazoult, avec des valeurs de $\mathrm{CI}_{50}$ de 8,88 $\pm 0,11 \mathrm{~g} / \mathrm{mL}$ et de 25,93 $\pm 0,16 \mathrm{~g} / \mathrm{mL}$, respectivement pour le bois et pour les feuilles. Cependant, la plus forte inhibition $(2,51 \pm 0,23 \mathrm{~g} / \mathrm{mL})$ occasionnée par l'HE de bois reste faible en comparaison avec celle de l'acide nordihydroguaiarétique $(0,61 \pm 0,02 \mathrm{~g} / \mathrm{mL})$ utilisé comme composé de référence. Par ailleurs, la xanthine oxydase est inhibée par ces $\mathrm{HE}$ avec des valeurs de $\mathrm{CI}_{50}$ allant de $6,54 \pm 0,25$ à $17,97 \pm 0,27 \mathrm{~g} / \mathrm{ml}$ et de $18,12 \pm 0,15$ à $29,4 \pm 0,19 \mathrm{~g} / \mathrm{mL}$, respectivement pour le bois et pour les feuilles. La plus forte inhibition $(6,54 \pm 0,25 \mathrm{~g} / \mathrm{mL})$ produite par l'HE de bois est significativement plus faible que celle de l'allopurinol $(2,45 \pm$ $0,19 \mathrm{~g} / \mathrm{ml}$ ) utilisé comme composé de référence (Djouahri et al., $2014 \mathrm{a}$ ).

Les résultats d'autres études sont colligés au tableau 4. 
Tableau 4 : Activité anti-inflammatoire de Tetraclinis articulata

\begin{tabular}{|c|c|c|c|}
\hline Région & $\begin{array}{l}\text { Partie } \\
\text { utilisée }\end{array}$ & Extrait/ H E & Modèle expérimental \\
\hline $\begin{array}{l}\text { Marrakech } \\
\text { Ait Aissi } \\
\text { Ihahane } \\
(30910 \mathrm{~N} \\
\text { et 09430 } \\
\text { O) } \\
\text { (El Jemli } \\
\text { et al., } \\
2016 \text { a) }\end{array}$ & Feuilles & $\begin{array}{l}\text { L'HE a été extraite } \\
\text { par hydro-distillation }\end{array}$ & $\begin{array}{l}\text { L'activité anti- } \\
\text { inflammatoire a été } \\
\text { évaluée à base de dosage } \\
\text { de l'œdème de la patte de } \\
\text { rat induit par le } \\
\text { carraghénane et par un } \\
\text { traumatisme après } \\
\text { injection de différentes } \\
\text { concentrations de l'HE de } \\
\text { T. articulata (100 et } 200 \\
\mathrm{mg} / \mathrm{kg}) \text {. }\end{array}$ \\
\hline
\end{tabular}

\begin{tabular}{|c|c|c|c|}
\hline $\begin{array}{l}\text { Ain Defla } \\
\text { (Algérie) } \\
\text { (200 km à } \\
\text { l'ouest) } \\
\text { (Djouahri } \\
\text { et al., }\end{array}$ & Feuille & $\begin{array}{l}\text { L'HE a été extraite } \\
\text { par hydro-distillation }\end{array}$ & $\begin{array}{l}\text { L'activité anti- } \\
\text { inflammatoire de l'HE a } \\
\text { été évaluée par le } \\
\text { pourcentage d'inhibition } \\
\text { de la 5-lipooxygénase et } \\
\text { de la xanthine oxydase }\end{array}$ \\
\hline
\end{tabular}

2016) de la xanthine oxydase

\begin{tabular}{|c|c|}
\hline Effet ou résultat & Principes actifs \\
\hline $\begin{array}{l}\text { 3h après l'administration } \\
\text { de la dose de } 200 \mathrm{mg} / \mathrm{kg} \\
\text { de l'HE, une réduction } \\
\text { efficace du gonflement a } \\
\text { été démontrée de } 68,42 \pm \\
8 \% \text { et de } 84,51 \pm 5,70 \% \\
\text { suite de traumatisme } \\
\text { chimique et mécanique, } \\
\text { respectivement, par } \\
\text { rapport aux groupes } \\
\text { témoin (eau distillée et } \\
\text { indométhacine) }\end{array}$ & $\begin{array}{l}\text { Les principaux } \\
\text { composés identifiés } \\
\text { dans l'analyse CG / SM } \\
\text { sont l'acétate de } \\
\text { bornyle }(26,81 \%), \text { le } \\
\text { camphre }(22,40 \%) \text { et } \\
\text { l' } \alpha \text {-pinène }(7,16 \%), \\
\text { avec } 25 \text { autres } \\
\text { constituants mineurs. }\end{array}$ \\
\hline $\begin{array}{l}\text { La 5-lipooxygénase et la } \\
\text { xanthine oxydase sont } \\
\text { efficacement inhibées par } \\
\text { l'HE avec des valeurs de } \\
\mathrm{CI}_{50} \text { de } 17,86 \pm 0,19 \\
\mu \mathrm{g} / \mathrm{mL} \text { et } 20,1 \pm 0,15 \mu \mathrm{g} / \\
\mathrm{mL} \text {, respectivement. } \\
\text { Cette activité d'inhibition } \\
\text { est faible à celle de }\end{array}$ & $\begin{array}{l}\text { Les composants } \\
\text { majeurs analysés par } \\
\text { CPG et CPG-SM sont : } \\
\text { l' } \alpha \text {-pinène }(29,0 \%) \text {, } \\
\text { l'acétate de bornyle } \\
(25,5 \%) \text {, le camphre } \\
(16,6 \%) \text { et le limonène } \\
(6,7 \%) \text { représentant } \\
96,6 \% \text { du total de l'HE. }\end{array}$ \\
\hline
\end{tabular}

l'inhibiteur spécifique

(NDGA) de la 5-

lipooxygénase $\left(\mathrm{CI}_{50}=\right.$

$0,6 \pm 0,001 \mu \mathrm{g} / \mathrm{mL}$ ) et

l'inhibiteur spécifique

(Allopurinol) de la

xanthine oxydase $\left(\mathrm{CI}_{50}=\right.$

$2,4 \pm 0,03 \mu \mathrm{g} / \mathrm{mL})$.

\begin{tabular}{llll}
\hline $\begin{array}{l}\text { Khemisset } \\
\text { (Maroc) }\end{array}$ & $\begin{array}{l}\text { Feuilles, } \\
\text { Rameaux }\end{array}$ & $\begin{array}{l}\text { L'HE a été extraite } \\
\text { par hydro-distillation }\end{array}$ & $\begin{array}{l}\text { L'activité anti- } \\
\text { inflammatoire a été } \\
\text { évaluée par la méthode }\end{array}$ \\
$\begin{array}{l}\text { (Bourkhiss } \\
\text { et } a l .,\end{array}$ & $\begin{array}{l}\text { Sciure du } \\
\text { 2010) }\end{array}$ & & $\begin{array}{l}\text { enzymatique de la 5- } \\
\text { lipoxygénase. }\end{array}$
\end{tabular}

Les trois parties de la

Les composants

plante montrent une

activité anti-

inflammatoire

considérable avec une

$\mathrm{CI}_{50}$ de 10,$71 ; 18,21$ et

$57,32 \mu \mathrm{g} / \mathrm{ml}$

respectivement pour le

tronc, les rameaux et les

feuilles, mais les trois

valeurs restent faibles en

comparaison avec le

NDGA ayant une $\mathrm{CI}_{50}$ de

$0,7 \mu \mathrm{g} / \mathrm{ml}$.

majeurs ont été analysés par la CPG et la CPG-SM. Ainsi, des feuilles et des rameaux sont principalement constitués de monoterpènes hydrocarbonés (respectivement 30,8\% et $55,53 \%$ ) et oxygénés (respectivement 60,9\% et $10,16 \%)$. Les rameaux sont relativement plus riches en sesquiterpènes (15,08\%).

\begin{tabular}{|c|c|c|c|c|}
\hline $\begin{array}{ll}\text { Oran } & \text { Feuilles } \\
\text { (Ouest de } & \\
\text { l'Algérie) } \\
\text { (Rached et } \\
\text { al., 2018) }\end{array}$ & $\begin{array}{l}\text { Une extraction par } \\
\text { partage liquide-liquide } \\
\text { a été effectuée pour } \\
\text { obtenir les fractions } \\
\text { organiques de l'extrait } \\
\text { aqueux en utilisant de } \\
\text { l'acétate d'éthyle et du } \\
\text { butanol, } \\
\text { successivement. }\end{array}$ & $\begin{array}{l}\text { L'activité anti- } \\
\text { inflammatoire a été } \\
\text { évaluée par la production } \\
\text { de NO par les lignées } \\
\text { cellulaires de } \\
\text { macrophages murins. }\end{array}$ & $\begin{array}{l}\text { À } 8 \mathrm{mg} / \mathrm{ml} \text {, l'extrait } \\
\text { aqueux brut, et les } \\
\text { fractions d'acétate } \\
\text { d'éthyle et de butanol } \\
\text { montrent une inhibition } \\
\text { significative de la } \\
\text { production de } \mathrm{NO} \text {, soit } \\
\mathrm{CE}_{50}=241 \pm 4 ; 130 \pm 9 \\
\text { et } 198 \pm 5 \mu \mathrm{g} / \mathrm{mL} \text {, } \\
\text { respectivement. }\end{array}$ & $\begin{array}{l}\text { Le profil des composés } \\
\text { phénoliques des } \\
\text { extraits a été obtenu } \\
\text { grâce à CL avec DMD } \\
\text { couplée à une IE en } \\
\text { tandem avec SM. } \\
\text { Ainsi, } 9 \text { composés ont } \\
\text { été identifiés : } \\
\text { (Epi)catéchine dimer } \\
\text { type B, (+)- catéchine, } \\
(-) \text {-Epi catéchine, } \\
\text { Myricétine-O- } \\
\text { pentoxyl- }\end{array}$ \\
\hline
\end{tabular}




\begin{tabular}{|c|c|c|c|c|}
\hline & & & & $\begin{array}{l}\text { O-hexoside, } \\
\text { Myricétine-3-O } \\
\text { rutinoside, Myricétine- } \\
\text { 3-O glucoside, } \\
\text { Myricétine-3-O } \\
\text { rhamnoside, } \\
\text { Quercétine-3-O } \\
\text { rhamnoside et } \\
\text { Kaempférol-3-O } \\
\text { rhamnoside. }\end{array}$ \\
\hline $\begin{array}{l}\text { Ain-Dalfa } \\
\text { localisée à } \\
200 \mathrm{~km} \text { à } \\
\text { l'ouest } \\
\text { d'Alger } \\
\text { (Algérie) } \\
\text { (Djouahri } \\
\text { et } \text { al., } \\
2014 \text { b) }\end{array}$ & $\begin{array}{l}\text { L'HE a été extraite } \\
\text { par hydro-distillation }\end{array}$ & $\begin{array}{l}\text { L'activité anti- } \\
\text { inflammatoire de l'HE a } \\
\text { été évaluée par le } \\
\text { pourcentage d'inhibition } \\
\text { de la lipooxygénase et de } \\
\text { la xanthine oxydase. }\end{array}$ & $\begin{array}{l}\text { La lipooxygénase et la } \\
\text { xanthine oxydase sont } \\
\text { efficacement inhibées par } \\
l^{\prime} \mathrm{HE} \text { avec des valeurs de } \\
\mathrm{CI}_{50} \text { de } 8,16 \pm 0,44 \\
\mu \mathrm{g} / \mathrm{mL} \text { et } 12,45 \pm 0,94 \mu \mathrm{g} \\
/ \mathrm{mL} \text {, respectivement. }\end{array}$ & $\begin{array}{l}\text { L'analyse de l'HE par } \\
\text { CPG, CPG/SM et } \\
\text { HPLC a montré qu'elle } \\
\text { est riche en } \\
\text { monoterpènes } \\
\text { oxygénés }(94,47 \%) \text { et } \\
\text { pauvre en } \\
\text { hydrocarbures } \\
\text { monoterpènes }(5,53 \%) \text {. } \\
\text { Les composants } \\
\text { majeurs sont l' } \alpha \\
\text { campholenal }(16,34 \%) \text {, } \\
\text { le trans-pinocarveol } \\
(15,45 \%), \text { le cis- } \\
\text { verbénol }(12,36 \%) \text { et } \\
\text { verbénone }(13,36 \%) \text {. }\end{array}$ \\
\hline
\end{tabular}

$\mathbf{C E}_{50}$ : concentration d'extrait correspondant à 50\% d'inhibition de la production d'oxyde nitrique (NO) (Rached et al., 2018); NDGA : acide nordihydroguaïaretique.

Antérieurement, il a été établi que les flavonoïdes sont dotés de propriétés anti-inflammatoires (Amezouar et al., 2013 ; Hmidani et al., 2019). Incontestablement, la myricétine-3-Orhamnoside, la catéchine et l'épicatéchine sont des flavonoïdes connus pour leur activité antiinflammatoire (Mamta et al., 2012 ; Tiwari et Husain, 2017 ; Rached et al., 2018). De tels composés sont présents dans les extraits du thuya de barbarie (Rached et al., 2018 ; Dane et al., 2015). D'ailleurs, il a été démontré que les flavonoïdes inhibent le processus inflammatoire in vivo et in vitro (Rached et al., 2018). En fait, ils fonctionnent en affectant les systèmes enzymatiques impliqués dans la génération de processus inflammatoire (Tiwari et Husain, 2017). Par ailleurs, il a été mis en évidence que l'HE de T. articulata inhibe la formation de l'œdème de la patte de rat induit par la carraghénine. Cette inhibition pourrait être due à la richesse de l'HE en terpènes tels que l' $\alpha$-pinène, le camphre, l'acétate de bornyle et le bornéol (El Jemli et al., 2016 a).

\subsubsection{Autres activités biologiques}

D'autres effets du T. articulata ont été mis en lumière par maintes investigations. Jlizi et al., (2018) montrent que la résine du thuya possède une activité antityrosinase avec 87,2\% 
d'inhibition à $50 \mu \mathrm{g} / \mathrm{mL}$ et qu'elle exerce un effet antitumoral significatif contre la lignée cellulaire d'adénocarcinome (HeLa) avec une concentration d'inhibition notable $\left(\mathrm{CI}_{50}=9,5 \pm\right.$ 1,0 $\mu \mathrm{g} / \mathrm{mL}$ ). L'HE de T. articulata aurait des effets similaires sur diverses lignées cellulaires cancéreuses humaines, comme celles du sein et de l'ovaire, avec une $\mathrm{CI}_{50}=80 \mu \mathrm{g} / \mathrm{mL}$ (Buhagiar et al., 1999). Il a été conclu, dans la même recherche, que les monoterpènes de l'HE sont efficaces pour induire l'apoptose (Buhagiar et al., 1999). Par ailleurs, Rached et al., (2018) ont conclu que l'activité antitumorale vis-à-vis de ces cellules cancéreuses est attribuée à la présence de flavonoïdes dans l'extrait à l'acétate d'éthyle de T. articulata. En effet, il a été mis en évidence que la catéchine, l'épicatéchine et la quercétine ont un bon effet sur l'inhibition de la prolifération de trois lignées cellulaires différentes du cancer de la prostate chez l'Homme (Rached et al., 2018). Parmi les mécanismes d'action: l'arrêt du cycle cellulaire, la régulation négative de la protéine mutante p53, l'inhibition de nombreuses enzymes déclenchant le cancer et l'expression des protéines Ras ont été rapportés (Tiwari et Husain, 2017).

Sadiki et al., (2018) ont montré que l'HE pourrait être un puissant agent pharmacologique contre la démence, dans la mesure où elle module l'activité cholinergique et favorise l'action antioxydante dans l'hippocampe des rats soumis à l'expérience.

D’autre part, il a été prouvé que la plante a également une activité larvicide. Effectivement, l'extrait aqueux de son bois présente des effets toxiques qui agissent au bout de 24 heures de l'exposition aux concentrations létales $\mathrm{CL}_{50}$ très faibles. L'expérience a été menée sur des larves de moustiques culicidés, à savoir: Culex pipiens (Linné), Aedes caspius (Pallas), Culiseta longiareolata (Aitken) et Anopheles maculipennis (Meigen) (Aouinty et al., 2006).

\subsection{Toxicité}

La plupart des études mentionnent que le thuya de barbarie n'est pas toxique (Zahir et al., 2020). Son innocuité a été mise en évidence chez les souris (Jemli et al., 2016 a) et les rats (Sadiki et al., 2018).

Plus intéressant encore est le résultat d'une investigation qui a démontré que l'extrait aqueux brut des feuilles de T. articulata de la région d'Oran (Algérie) ne présente pas de cytotoxicité à l'égard des cellules primaires du foie de porc, avec une CI 50 de la croissance cellulaire supérieure à $400 \mu \mathrm{g} / \mathrm{mL}$, en comparaison avec l'éllipticine qui a une $\mathrm{CI}_{50}$ de l'ordre de 3,2 $\mu \mathrm{g} / \mathrm{mL}$ (Rached et al., 2018). 
Néanmoins, face à tous ces résultats optimistes, un premier cas clinique d'eczéma de contact a été rapporté après l'application unique d'une poudre de feuilles et de rameaux séchés de $T$. articulata et solubilisée dans l'eau à température ambiante. Selon la même étude, ce risque de toxidermie pourrait être dû à un ou à plusieurs composants majoritaires, et potentiellement sensibilisants, des feuilles et des rameaux de la plante, notamment le limonène, l' $\alpha$-pinène et le camphre (Zahir et Rahmani, 2020).

D'autre part, la plupart des études ne mesurent actuellement que la toxicité aiguë. Cependant, la toxicité est un terme relatif qui varie selon la partie de la plante d'où a été puisé l'extrait, les quantités consommées et la durée de cette consommation. Par conséquent, il est essentiel de tester la toxicité de l'extrait brut, de l'HE et des composés individuels ou associés afin de fournir des informations fiables sur les réactions indésirables pouvant résulter éventuellement d'une exposition prolongée à l'utilisation d'extraits ou de l'huile du thuya (Azzi et al., 2012). Par ailleurs, du point de vue environnemental, il est jugé également nécessaire de mener des études écotoxicologiques pour évaluer les toxicités aiguës et chroniques potentielles de l'extrait ou de l'HE de $T$. articulata sur les espèces-clés des écosystèmes aquatiques et terrestres. Montasir et al., (2017) signalent en effet que l'utilisation des extraits aqueux du thuya pour leurs activités larvicides contre les moustiques peut générer une toxicité aiguë et de reprotoxicité, sur une population non ciblée telle que celle de Daphnia magna. Cette espèce peut jouer un rôle important dans la chaîne alimentaire au niveau des écosystèmes aquatiques marocains.

\section{Conclusion}

La présente revue de synthèse a ciblé le recensement de l'ensemble des activités biologiques de la plante $T$. articulata, et ce, à partir de l'analyse de plusieurs recherches scientifiques menées au Maghreb et en Égypte.

Ainsi, il a été mis en évidence que cette essence forestière est dotée d'une activité antifongique contre une gamme de phytopathogènes suggérant que les HE du thuya seraient des biopesticides alternatifs des pesticides chimiques.

Par ailleurs, la plante possède également une activité antibactérienne contre un ensemble de bactéries pathogènes présentant une résistance aux antibiotiques. De la sorte, l'exploitation des extraits ou de l'HE de T. articulata peut constituer une voie alternative prometteuse pour développer éventuellement de nouvelles préparations à effet antibactérien. 
Il a été rapporté également que le thuya de barbarie est doté des activités anti-inflammatoires, antioxydantes et antitumorales.

Aux propriétés qui viennent d'être décrites devraient s'ajouter les résultats d'autres investigations toxicologiques complémentaires et qui pourraient juger l'innocuité de la plante avant d'être envisagée dans le domaine médical et agricole. De plus une recherche sur les modes d'action des molécules actives à l'échelle cellulaire permettrait de comprendre plus complètement l'intérêt des utilisations traditionnelles et modernes de cette essence forestière.

\section{Références}

ABI-Ayad F.Z., ABI-Ayad M., Lazzounia H.A., Rebiahib S.A., Bessierec. 2011. Antibacterial activity of essential oil extracted from leaves of Tetraclinis articulata (Vahl) Masters from Algeria flora, Scholars Research Library, 1-6 p.

Agence Nationale de Sécurité Sanitaire de L'alimentation de L'environnement et du Travail. 2016 : Avis de l'Anses relatif à l'évaluation des substances inscrites au programme de travail 2015 de l'Agence dans le cadre de la Stratégie nationale sur les perturbateurs endocriniens. Disponible en ligne : https://www.actu-environnement.com/media/pdf/news-26943AvisAnsesPE2015.pdf (consulté le 4 juillet 2020).

Akbli M., Rhallabi N., Ait Mhand R., Akssira M., Mellouki F. 2016. Activité antibactérienne de l'huile essentielle de la sciure du bois de loupe de Tetraclinis articulata (Vahl) master du Maroc sur des souches d'origine clinique. International Journal of Innovation and Applied Studies, $16(2): 314-321$.

Amezouar F., Badri W., Hsaine M., Bourhim N., Fougrach H. 2012. Evaluation des activités antioxydante et anti-inflammatoire de Erica arborea L. du Maroc. Pathologie Biologie, 61 : 254-258.

Aouinty B., Oufara S., Mellouki F., Mahari S. 2006. Évaluation préliminaire de l'activité larvicide des extraits aqueux des feuilles du ricin (Ricinus communis L.) et du bois de thuya (Tetraclinis articulata (Vahl) Mast.) sur les larves de quatre moustiques culicidés : Culex pipiens (Linné), Aedes caspius (Pallas), Culiseta longiareolata (Aitken) et Anopheles maculipennis (Meigen). Biotechnol. Agron. Soc. Environ. 10 (2), 67 - 71.

Azzi R., Djaziri R., Lahfa F., Sekkal FZ., Benmehdi H., Belkacem N. 2012. Ethnopharmacological survey of medicinal plants used in the traditional treatment of diabetes mellitus in the North Western and South Western Algeria. Journal of Medicinal Plants Research. 6 (10) ; 2041-2050.

Ben Jemia M., Chaabane S., Senatore F., Bruno M., Elyes Kchouk M. 2013. Studies on the antioxidant activity of the essential oil and extract of Tunisian Tetraclinis articulata (Vahl) Mast. (Cupressaceae). Natural Product Research : Formerly Natural Product Letters, 27(16) : 1419-1430. DOI : https://doi.org/10.1080/14786419.2012.717289 
Ben Ghnaya A., Amri I., Hanana M., Gargouri S., Jamoussi B., Romanef A., Hamrouni L. 2016. Tetraclinis articulata (Vahl.) Masters essential oil from Tunisia: Chemical characterization and herbicidal and antifungal activities assessment, Campus Universitaire.113-117p.

Bourkhis M., Hnach M., Bourkhis B., Ouhssine M. Chaouch A., 2007. Composition chimique et propriété antimicrobienne de l'huile essentielle extraite des feuilles de Tetraclinis articulata Vahl du Maroc. Afrique Sci., 3 (2), 232-242.

Bourkhiss M., Hnach M., Paolini J. et Costa J., 2010. Propriétés antioxydantes et antiinflammatoires des huiles essentielles des différentes parties de Tetraclinis articulata (vahl) masters du Maroc, Bulletin de la Société Royale des Sciences de Liège, 79. 141 - 154.

Bouyahya, A., Lagrouh, F., El Omari, N., Bourais, I., El Jemli, M., Marmouzi, I., Salhi, N., El Abbes, F.M., Belmehdi, O., Dakka, N., Bakri, Y. 2019. Essential oils of Mentha viridis rich phenolic compounds show important antioxidant, antidiabetic, dermatoprotective, antidermatophyte and antibacterial properties, Biocatalysis and Agricultural Biotechnology, 26 pages. DOI : https://doi.org/10.1016/j.bcab.2019.101471

Buhagiar J.A., Podesta M.T., Wilson A.P., Micallef M.J., Ali S. 1999. The induction of apoptosis in human melanoma, breast and ovarian cancer cell lines using an essential oil extract from the conifer Tetraclinis articulata. Anticancer Res. 19(6B) : 5435-5543.

Chikhoune A., Hazzit M., Kerbouche L., Baaliouamer A., Aissat K. 2013. Tetraclinis articulata (Vahl) Masters essential oils : chemical composition and biological activities. Journal of Essential Oil Research, DOI:10.1080/10412905.2013.774625.

Chraibi M, Farah A, Elamin O, Iraqui HM, Benbrahim KF. 2020. Characterization, antioxidant, antimycobacterial, antimicrobial effects of Moroccan rosemary essential oil biological activities and synergistic effect with carvacrol, Journal of Advanced Pharmaceutical Technology \& Research. $11: 25-29$.

Dane Y., Mouhouche F., Canela-Garayoa R., Delpino-Rius A. 2015. Phytochemical Analysis of Methanolic Extracts of Artemisia absinthium L. 1753 (Asteraceae), Juniperus phoenicea L., and Tetraclinis articulata (Vahl) Mast, 1892 (Cupressaceae) and evaluation of their biological activity for stored grain protection. Arab J Sci Eng, 1-12. DOI : https://doi.org/10.1007/s13369-015-1977-2

Djouahri A., Boualema S., Boudarenea L., Baaliouamer A. 2014 a. Geographic's variation impact on chemical composition, antioxidant and anti-inflammatory activities of essential oils from wood and leaves of Tetraclinis articulata (Vahl) Masters. Ind. Crops Prod, 63 : 138-146. DOI : http://dx.doi.org/10.1016/j.indcrop.2014.10.018 
Djouahri A., Sakaa B., Boudarenea L., Benseradj F., Aberranea S., Aitmoussaa S., Chelghoum, Lamari CL., Sabaouc N., Baaliouamer A. 2014 b. In vitro synergistic/antagonistic antibacterial and anti-inflammatory effect of various extracts/essential oil from cones of Tetraclinis articulata (Vahl) Masters with antibiotic and anti-inflammatory agents, Industrial Crops and Products, 56: 60-66.

Djouahri A., Sebiane S., Kellou F., Lamari L., Sabaou N., Baaliouamer A., Boudarene L, 2016. Inhibitory effect on corrosion of carbon steel in acidic media, antioxidant, antimicrobial, anti-5- lipooxygenase and anti-xanthine oxidase activities of essential oil from Tetraclinis articulata (Vahl) Masters leaves. Journal of Essential Oil Research.1-9 p.

El Jemli M., Kamal R., Marmouzi I., Doukkali Bouidida EH, Touati D, et al., 2016 a. Chemical composition, acute toxicity, antioxidant and anti-inflammatory activities of Moroccan Tetraclinis articulata L. J Tradit Complement Med; 7(3): 281-287.

El Jemli M., Kamal R., Marmouzi I., Zerrouki A., Cherrah Y., Alaoui K. 2016 b. RadicalScavenging Activity and Ferric Reducing Ability of Juniperus thurifera (L.), J. oxycedrus (L.), J. phoenicea (L.) and Tetraclinis articulate (L.). Advances in Pharmacological Sciences, 1-7. DOI : http://dx.doi.org/10.1155/2016/6392656.

El Moussaouiti M., Talbaoui A., Gmouh S., Aberchane M., Benjouad A., Bakri Y, D. Pascal Kamdem. 2010. Chemical composition and bactericidal evaluation of essential oil of Tetraclinis articulata burl wood from Morocco. J Indian Acad Wood Sci, 7(1-2) : 14-18.

DOI : http://dx.doi.org/10.1007/s13196-010-0003-2

El Omari N., Sayah K., Fettach S., El Blidi O., Bouyahya A., My El Abbes F., Kamal R., Barkiyou M. 2019. Evaluation of In Vitro Antioxidant and Antidiabetic Activities of Aristolochia longa Extracts. Evidence-Based Complementary and Alternative Medicine, Article ID 7384735, 9 pages. DOI : https://doi.org/10.1155/2019/7384735

Fidah A., Rahouti M., Kabouchi B., Famiri A.., 2019. Chapter : Durable Woods and Antifungal Activity of Their Essential Oils: Case of Tetraclinis articulata (Vahl) Masters and Cedrus atlantica Manetti in Essential Oils - Oils of Nature, Intechopen Ed.). 12p. DOI : http://dx.doi.org/10.5772/intechopen.87214

Hadjadj K., Letreuch Belarouci A., 2017 : Synthèse bibliographique sur le thuya de berbérie [Tetraclinis articulata (Vahl) Mast.]. Geo-Eco-Trop. 41 (1) : 13-27.

Herzi N., Bouajila J., Camy S., Romdhane M., Condoret J. 2013. Comparison of different methods for extraction from Tetraclinis articulata: Yield, chemical composition and antioxidant activity. Food Chemistry, 141 (4) : 3537-3545.

Hmidani A., Bouhlali EDT, Khouya T., Ramchoun M., Filali-Zegzouti Y, Alem C., Benlyas M. 2019. Antioxidant, anti-inflammatory and anticoagulant activities of three Thymus species grown in southeastern Morocco. Future Journal of Pharmaceutical Sciences 5:4. DOI : https://doi.org/10.1186/s43094-019-0005-x 
Ibrahim T, El-Helac A, El-Hefnawy H., Al-Taweel A et Perveena S., 2017. Chemical Composition and Antimicrobial Activities of Essential Oils of Some Coniferous Plants Cultivated in Egypt. Iranian Journal of Pharmaceutical Research, 16(1) : 328-337.

Jlizi S., Zardi-Bergaouia A., Znatia M., Flamini G., Ascrizzi R., Ben Jannet H., 2018. Chemical composition and biological evaluation of the resin from Tetraclinis articulata (Vahl.) Masters : A promising source of bioactive secondary metabolites, Industrial Crops \& Products $124: 74-83$.

Mamta S., Jyoti S., Alka P. 2012. Flavonoids and phenolic acids as antioxidants in plants and human health. International Journal of Pharmaceutical Sciences Review and Research. 16(2) : 130-134.

Mhirit O., Benchekroun F., 2006. Les écosystèmes forestiers et péri-forestiers : situation, enjeux et perspectives pour 2025, Contribution au Rapport sur le Développement Humain (RDH50) Maroc, GT8-7, 397-483.

Montassir L., Berrebaan I., Mellouki F., Zkhiri F., Boughribil S., Bessi H. 2017. Acute toxicity and reprotoxicity of aqueous extract of a Moroccan plant (Tetraclinis articulata) on freshwater cladoceran Daphnia magna. Journal of Materials and Environmental Sciences. $8(2): 770-776$.

Rached W., Zeghadab F., Bennaceurb M., Barrosa L., Calhelhaa R., Helenoa S., Alvesa M., Carvalhoa A., Maroufe A., Ferreiraa I. 2018. Phytochemical analysis and assessment of antioxidant, antimicrobial, antiinflammatory and cytotoxic properties of Tetraclinis articulata (Vahl) Masters leaves. Industrial corps \& product. 112:460-466.

Sadiki F., El Idrissi M., Cioanca O., Trifan A., Hancianu M., Hritcu L., Postu PA. 2018. Tetraclinis articulata essential oil mitigates cognitive deficits and brain oxidative stress in an Alzheimer's disease amyloidosis model, Phytomedicine, 1-15. DOI : https://doi.org/10.1016/j.phymed.2018.10.032.

Sayah K., Marmouzi I., Naceiri Mrabti H., Cherrah Y., My El Abbes F. 2017. Antioxidant Activity and Inhibitory Potential of Cistus salviifolius (L.) and Cistus monspeliensis (L.) Aerial Parts Extracts against Key Enzymes Linked to Hyperglycemia. BioMed Research International, Article ID 2789482, 7 pages.

Talbaoui A., El Hamdaoui L., El Moussaouiti M., Aneb M., Amzazi S., Bakri Y., 2016. GCMS analysis and antibacterial activity of hydro-distillation oil from Tetraclinis articulata wood grown in Khemisset (Morocco). J Indian Acad Wood Sci, 1-4 p.

Tekaya-Karoui A., Boughalleb N., Hammami S., Ben Jannet H., Mighri Z. 2011. Chemical composition and antifungal activity of volatile components from woody terminal branches and roots of Tetraclinis articulata (Vahl.) Masters growing in Tunisia. African Journal of Plant Science. 5(2) : 115-122.

Tiwari S C., Husain N. 2017. Biological activities and role of flavonoids in human health-a review. Indian J. Sci. Res. 12 (2) : 193-196. 
Valko M., Leibfritz D., Moncol J., Cronin M. T. D., Mazur M., Telser J. 2007. Free radicals and antioxidants in normal physiological functions and human disease," International Journal of Biochemistry and Cell Biology. 39 (1) : 44-84.

Zahir I., Houari A., Iraqui M., Ibnsouda S. 2018 a. Valorisation de l'activité antibactérienne des microorganismes isolés à partir des biotopes marocains et caractérisation partielle de leurs principes actifs. Proceedings BIOSUNE'1 - 2018. 80-95. ISBN : 978-9920-37-054-7.

Zahir I., Babouchi M., Boulanour H., El Louyti M. 2018 b. Effet des microorganismes isolés à partir des biotopes marocains sur les phytopathogènes : revue bibliographique. Revue Agrobiologia. 8 (2) : 971-983.

Zahir I. 2016. Effect of Moroccan plants against phytopathogenic microorganisms : a review. British Biotechnology Journal. 10 (1) : 1-36.

Zahir I., Rahmani A. 2020. Premier cas clinique d'eczéma de contact causé par Tetraclinis articulata. International Journal of Innovation and Applied Studies, 28 (2) : 342-346.

Zahir I., Elazaoui S., Chakouri M., Naouer B. 2020. Etude ethnobotanique de Tetraclinis articulata dans la région de Béni Mellal - Khénifra. Ethnobotany Research \& Applications, $19(36): 1-22$. 